\title{
1. Objektivität und Standortgebundenheit
}

Wer sich zu historischer Objektivität äußern möchte, sieht sich einer extrem heterogenen Begriffslandschaft und einer schier unüberschaubaren Fülle an einschlägigen Beiträgen aus mehreren Fachdisziplinen ${ }^{9}$ gegenüber. Bevor daher die angestrebte ausgewogene Einschätzung zu historischer Objektivität vorgelegt werden kann, muss die Schwierigkeit gemeistert werden, dass nicht ein Objektivitätsbegriff zur Diskussion steht, sondern eine Reihe von alternativen Objektivitätsbegriffen in den verschiedensten Kontexten kursieren, deren Beziehungen und Interdependenzen auf das mannigfaltigste konzeptualisiert worden sind. Eine verwendbare Konzeption von historischer Objektivität herausarbeiten zu können, setzt daher voraus, sich über die diversen Objektivitätsbegriffe einen Überblick zu verschaffen und sie nach Möglichkeit zu ordnen (zumindest soweit es für die vorliegende Untersuchung von Interesse ist). Aufgrund der unübersichtlichen Diskussionslage und der schieren Menge an Beiträgen wird im folgenden nicht versucht, eine vollständige Evaluation aller im Umlauf befindlichen Beiträge zu absolvieren. An Stelle eines solchen tour d'horizon wird versucht, Gattungen und Kategorien zu entwickeln, mit deren Hilfe die diversen Beiträge erfasst und argumentativ integriert werden können.

$\mathrm{Zu}$ diesem Zweck wird im ersten Kapitel ein „Komponentenmodell“ historischer Objektivität eingeführt werden, das die begriffliche Explikation des Objektivitätsbegriffs an die Hand geben wird, auf deren Basis die anvisierte ausgewogene Konzeption historischer Objektivität erörtert werden kann. Erst wenn einige begriffliche Klarheit erreicht worden ist, lassen sich die beiden Extreme der postmodernen Objektivitätsverabschiedung und der positivistischen Objektivitätsverengung einordnen, und erst dann lässt sich

9 Ein erster Eindruck über die Bandbreite der an der Objektivitätsdiskussion beteiligten Fachdisziplinen lässt sich gewinnen, betrachtet man die jeweilige fachdisziplinäre Beheimatung der Beiträge in Megill 1994a. 
der hier zu machende Vorschlag zwischen diesen beiden Extremen hindurchnavigieren. Zuallererst jedoch bedarf die Untersuchung der Begriffslandschaft einer vorgängigen Eingrenzung.

\subsection{Eingrenzung des Untersuchungsbereichs}

„Objektivität“" wird bekanntermaßen in unterschiedlichsten Kontexten verwendet. Die alltäglichen wie die diversen fachspezifischen Redeweisen von „objektiv“/,Objektivität“ verwenden diesen Begriff um „objektive Richter“, die „objektive Urteile“ gefällt haben, zu loben; um einen Sachverhalt als „objektiv bestehend“ zu verteidigen; um Abstrakta wie Werten oder Normen eine „objektive Existenz“ zuzusprechen oder um „objektive Erkenntnis“ vor „subjektiver Meinung“ auszuzeichnen, wobei letztere anhand ihres Verstoßes gegen „objektive Methoden“ als „subjektiv“ ausgewiesen wird. Werte, Methoden, Erkenntnisse, Personen, Urteile, Sachverhalte, Gegenstände, Prozesse, Ereignisse u. a. m. werden als objektiv bezeichnet. Weil nicht alle diese potentiellen Objektivitätsträger Gegenstand einer Untersuchung zu historischer Objektivität zu sein brauchen, liegt es auf der Hand, einige dieser Verwendungsweisen des Objektivitätsbegriffs aus der folgenden Untersuchung herauszuhalten. Zu diesem Zweck soll zunächst eine grobe Klassifikation verschiedener Objektivitätsauffassungen vorgenommen werden.

Eine Klassifikation von Objektivitätsauffassungen beginnt am besten über einen Vorstellungskern von „Objektivität“/,objektiv“, dem man sich am ehesten über den Gegenbegriff zu Objektivität, die Subjektivität, nähert. Objektivität ist über die Vorstellung der Unabhängigkeit von subjektiven Einflüssen bestimmt. Was auch immer als objektiv ausgezeichnet werden soll, soll im Gegensatz zu einer (in der Regel unerwünschten) subjektiven Beeinflussung ausgezeichnet werden. Ein objektiver Richter richtet unabhängig von den Einflüssen der Streitparteien; ein objektiver Wert ,existiert` 
unabhängig davon, ob die ihm Unterworfenen ihn teilen oder ihn ablehnen; auch Methoden sind nur dann objektiv, wenn sie anwendbar sind, ohne der subjektiven Willkür einen Beeinflussungsspielraum einzuräumen.

Diese erste Annäherung gegeben, kann weiter die Frage gestellt werden, was objektiv ist. Darauf lassen sich zwei Arten von Antworten geben, je nachdem, wie die Frage aufgefasst wird. Zum einen kann diese Frage als Frage danach verstanden werden, welche Gegenstandsklassen objektiv sein können - es handelt sich um die Frage nach der attributiven Verwendung von ,objektiv“. Richter bei Gericht, Schiedsrichter bei Sportveranstaltungen und Wissenschaftler können für den allgemeinen Sprachgebrauch objektiv sein, gleichfalls Methoden, Messungen und Prozeduren, Erkenntnisse und Darstellungen von Erkenntnissen wie auch abstrakte ${ }^{10}$ Gegenstände. Man könnte anhand dieser Auflistung davon sprechen, dass auf Personen, Methoden, Gehalte epistemisch-doxastischer Zustände und (abstrakte) Gegenstände „objektiv“ attributiv verwendet werden kann.

Zum anderen kann die Frage als eine definitorische Was-ist-Frage aufgefasst werden. Die Antworten auf diese Frage sind Vorschläge dazu, was mit dem Begriff „Objektivität“ gemeint ist. Meist handelt es um die Angabe einer Eigenschaft, aufgrund derer die attributive Verwendungsweise von „objektiv" begründet werden kann, wobei diese Eigenschaft dann als definitionale Eigenschaft von Objektivität gelten kann, weil sie spezifiziert, von welcher Art subjektivem Einfluss der Gegenstand frei ist. Von einer objektiven Person wird gesprochen, wenn sie die Eigenschaft der Unparteilichkeit besitzt; eine Methode ist dann objektiv, wenn sie bestimmte prozedurale Eigenschaften besitzt, die eine subjektive Einflussnahme ausschließen; Gehalte epistemisch-doxastischer Zustände werden dann als objektiv bezeichnet, wenn sie abbilden, ,wie die Dinge liegen' oder ,sind', unabhängig davon, was ein subjektiver Eindruck vorgaukeln mag; und wenn ein (abstrakter) Gegenstand, zum Beispiel ein Sachverhalt oder ein Wert, objektiv genannt wird, dann wird damit gesagt, dass er die ,Eigenschaft ${ }^{\star}$ der

10 Von materiellen Gegenständen zu sagen, sie seien objektiv, wirkt pleonastisch. Von einem Stein, der auf dem Weg liegt, zu sagen, er existiere objektiv, dürfte ziemliches Befremden auslösen, es sei denn, damit soll emphatisch einer idealistischen These bezüglich der materiellen Nicht-Existenz des Steins widersprochen werden. 
Existenz unabhängig von irgendwelchen Subjekten oder subjektiven Einstellungen besitzt. Man kann in diesen Fällen von vier Objektivitäten sprechen: Objektivität als Unparteilichkeit, prozedurale oder methodologische Objektivität, absolute oder epistemologische Objektivität und ontologische Objektivität. ${ }^{11}$

Doch damit sind die gängigen Formen der Objektivität noch nicht erschöpft: Die ontologische Objektivität besitzt dann eine besondere, moralische Dimension, wenn einem Wert objektive Existenz zugesprochen wird. In diesem Fall kann davon gesprochen werden, dass moralische Objektivität vorliegt: Wenn ein Wert bzw. eine Norm existiert, gilt sie objektiv. Weiter wird immer wieder die methodologische wie auch die epistemologische Objektivität mit Intersubjektivität in Verbindung gebracht: ${ }^{12}$ Methoden seien dann objektiv, wenn sie intersubjektiv gültig sind, wie auch Erkenntnisse objektiv seien, wenn sie intersubjektiv überprüfbar sind. Darüber hinaus liegt eine weitere Objektivitätsvorstellung mit der konsensualen Objektivität vor, die wiederum sowohl an den Methoden als auch an den Erkenntnissen ansetzt. Objektiv ist eine Methode dann, wenn sie von einem fachdisziplinären Konsens als solche anerkannt wird. Gleiches gilt für die Objektivität von Erkenntnis: Erkenntnis ist dann objektiv, wenn sie als solche von einem fachdisziplinären Konsens anerkannt wird. Damit treten zu den vier genannten Objektivitäten drei weitere hinzu, woraus sich die folgende Reihe von gängigen Objektivitätskonzeptionen ergibt: Objektivität als Unparteilichkeit, prozedurale (methodologische) Objektivität, absolute (epistemologische) Objektivität, ontologische Objektivität, moralische Objektivität, Objektivität als Intersubjektivität und konsensuale Objektivität.

Ausgehend von der thematischen Ausrichtung der vorliegenden Arbeit kann eine erste dieser sieben Objektivitätsvorstellungen aus der folgenden Diskussion ausgeschlossen werden. Moralische Objektivität soll im folgen-

11 Vgl. die Klassifikation nach epistemologischer, moralischer, ontologischer und methodologischer Objektivität in Daston/Railton 2007, 56 und die Megills (1994b) nach prozeduraler, absoluter, dialektischer und disziplinärer Objektivität. Die Terminologien werden hier eklektisch übernommen, wenn auch eine differenziertere Klassifikation eingeführt wird.

12 Agazzi 1988 etwa betrachtet Intersubjektivität als eine der beiden verbreitetsten Objektivitätsauffassungen (ebd., 18). 
den nicht mehr thematisiert werden, weil die Frage nach der Existenz bzw. absoluten Geltung von Werten und Normen in einer wissenschaftstheoretisch-epistemologischen Arbeit nicht relevant ist, zumindest nicht in dem Sinn, dass sie danach fragen muss, ob Werte bzw. Normen unabhängig von Subjekten existieren oder ob sie nur konventionell gelten.

Neben der moralischen Objektivität soll aber auch noch ontologische Objektivität in den folgenden Betrachtungen außen vor bleiben. Die ontologische Fragestellung nach der Existenz der empirischen Realität verengt sich in einer Arbeit zu historischer Objektivität auf die Frage nach der Existenz vergangener Wirklichkeit. Im folgenden soll diese Frage als in einem Common-sense-Sinn beantwortet gelten. So wie das gerade zurückliegende Frühstück und der Kinobesuch am vergangenen Samstag objektiv existierende Ereignisse für jedermann sind, so sollen historische Ereignisse als objektiv existierend angesehen werden. Das bedeutet vor allem, Argumentationen, die einem historischen Idealismus das Wort reden wollen, nicht zu diskutieren. Dieser Common-sense-Realismus ist der kleinste gemeinsame Nenner aller folgenden Positionen (und auch der beinahe der gesamten wissenschafts- oder erkenntnistheoretischen Literatur zum The$\left.\mathrm{ma}^{13}\right)$.

Zuletzt soll noch Objektivität als Intersubjektivität aus den folgenden Argumentationen herausgehalten werden. Dafür müssen knapp einige Differenzierungen vorgenommen werden. Zum einen kann sich Intersubjektivität auf zwei Gegenstandsklassen beziehen: (A) intersubjektive Methoden und (B) intersubjektive Erkenntnisse. Zu anderen kann ,intersubjektiv“ auf zweierlei Weise verstanden werden: (1) als intersubjektive Überprüfbarkeit, das heißt als intersubjektiver Zugang zu (nach A:) Methoden oder (nach B:) Erkenntnissen zum Zwecke einer epistemologischen Einschätzung und (2) als intersubjektive Geltung von (nach A:) Methoden oder (nach B:) Erkenntnissen. Aus dieser Differenzierung ergeben sich verschiedene Kombinationsmöglichkeiten dahingehend, was unter Objektivität als Intersubjektivität verstanden werden kann. Geht man sie der Reihe nach durch, stellt sich heraus, dass keine eine Bereicherung der folgenden Dis-

13 Mit der Ausnahme einiger postmoderner Autoren, die hier keine Rolle spielen werden (vgl. Abs. 2.1). 
kussion mit sich bringt.

Die Kombinationen (A1) und (B1) können gleichzeitig behandelt werden, weil die Auffassung, Objektivität sei als intersubjektiver Zugang zu Methoden oder Erkenntnissen zum Zweck ihrer Überprüfung durch die Erkenntnissubjekte zu begreifen, in beiden Fällen trivial ist. Dass sowohl die Methoden des Historikers als auch die Ergebnisse, die er mittels dieser Methoden zutage fördert, in dem Sinn intersubjektiv sein müssen, dass sie möglichst jederzeit allen anderen Erkenntnissubjekten zur Überprüfung zugänglich sein müssen, ergibt sich bereits aus dem rein pragmatischen Grund, dass er, will er seine Ergebnisse von der Fachwelt anerkannt haben, er sie wie auch seine Methoden so formulieren und wählen muss, dass sie seinen Kollegen und den „interessierten Laien“ für eine wissenschaftliche Einschätzung zugänglich sein müssen. Diese ubiquitäre Rolle von Intersubjektivität macht sie $\mathrm{zu}$ einem ständigen Begleiter historischer Objektivität und der fachlichen Auseinandersetzung um sie, ihr Beitrag zur wissenschaftlichen Erkenntnis ist aber ähnlich unspezifisch wie etwa der Beitrag der Sprache oder der logischer Gesetze zu wissenschaftlicher Erkenntnis. Methodologische, empirische oder hermeneutische Diskussionen können überhaupt erst geführt werden, wenn gewisse Standards der Intersubjektivität eingehalten werden. Diese Standards sind auf beinahe allen Ebenen der historischen Forschung unabdingbar. ${ }^{14}$ Jeder Schritt der historiographischen Forschung muss (zumindest prinzipiell) anderen Teilnehmern des historischen Diskurses transparent gemacht werden können - und Transparenz bedeutet intersubjektive Zugänglichkeit und Überprüfbarkeit. Intersubjektivität in diesem Sinn ist eine Vorbedingung, die erfüllt sein muss, bevor überhaupt erst epistemologische Fragen danach erhoben werden können, ob historische Darstellungen bzw. die Methoden, die zu ihnen geführt haben, objektiv sind.

Gerade dieser eigentlich wissenschaftlich-epistemologischen Frage nach der Geltung von Erkenntnis resp. von Methoden gehen die Kombinationsmöglichkeiten (A2) und (B2) nach. Objektivität als Intersubjektivität meint

14 Aus diesem Grund greift auch die Verortung der Intersubjektivität bei Douglas 2004, 463 zu kurz. Sie sieht lediglich im Rahmen des Ergebniskonsensus Intersubjektivität vor. 
dann die intersubjektive Geltung der Methoden oder Erkenntnisse. Was könnte damit gemeint sein, dass bestimmte Erkenntnisse intersubjektiv gelten (B2)? Damit ist entweder gemeint, dass Erkenntnisse gelten, weil ihnen die Erkenntnissubjekte zustimmen oder bestimmte Erkenntnisse gelten, weil sie das korrekt erfassen, was sich in der Vergangenheit zugetragen hat und ihnen deshalb die Erkenntnissubjekte zustimmen. In keinem der beiden Fälle trägt (B2) etwas zur Diskussion bei, das nicht auch in einer der anderen Objektivitätsauffassungen beschlossen liegt. Die Zustimmung der Erkenntnissubjekte zum jeweiligen Forschungsergebnis ist das Spezifikum der konsensualen Objektivität - und wird entsprechend an dieser Stelle diskutiert -, während sich das korrekte Erfassen dessen, was sich in der Vergangenheit zugetragen hat, auf die Korrespondenz von Darstellung mit dem Dargestellten und damit auf das, was als epistemologische bzw. absolute Objektivität bereits eingeführt worden ist, reduziert und entsprechend in diesem Zusammenhang diskutiert werden wird.

Bleibt noch die Frage, ob wenigstens von (A2), der intersubjektiven Geltung von Methoden, ein eigenständiger Beitrag zur Diskussion um historische Objektivität zu erwarten ist. Auch in dieser Lesart kann die Frage verneint werden. Sollte die Geltung von Methoden daran hängen, dass eine Gruppe von Historikern von ihrer Richtigkeit überzeugt ist, fällt diese Diskussion wieder in den Bereich der konsensualen Objektivität. Sollte damit aber gemeint sein, dass Methoden dann als intersubjektiv gültig betrachtet werden sollen, wenn sie Mittel dazu waren, wissenschaftlich korrekte Ergebnisse zu erzielen, dann trägt eine Antwort auf die Frage, ob eine Methode intersubjektiv gilt, nichts bei, was nicht schon bei der Antwort auf die Frage nach der Objektivität der Methoden beantwortet werden soll. In diesem Fall rückt die methodologische Objektivität in den Vordergrund.

Die folgenden Untersuchungen sind damit zunächst einmal auf eine Diskussion von vier Objektivitätsauffassungen eingegrenzt worden: der konsensualen, der methodologischen (prozeduralen), der epistemologischen (absoluten) Objektivität und der der Objektivität als Unparteilichkeit. Jede dieser Objektivitätsauffassungen spielt in irgendeiner Form in der einschlägigen Literatur eine gewisse Rolle.

Die Schwierigkeit im Umgang mit (historischer) Objektivität, trotz die- 
ser ersten Einschränkung des Untersuchungsbereichs mit einer recht unübersichtlichen Begriffslandschaft zurecht kommen $\mathrm{zu}$ müssen, besteht aber weiterhin. Und solange die umlaufenden Objektivitätsauffassungen lediglich klassifikatorisch aufgezählt und in diesem Zuge die Diskussion um nur einige Begriffsauffassungen entlastet worden ist, kann eine fokussierte Behandlung des Themas nicht geleistet werden. Um aber zur angekündigten ausgewogenen Einschätzung davon kommen zu können, in welchem Sinn und bis zu welchem Grad historische Objektivität möglich ist, muss ein Modell vorgelegt werden, das es ermöglicht, die folgende Diskussion auf einen zentralen Diskussionsstrang zu fokussieren, ohne damit den häufig hervorgerufenen Eindruck zu erwecken, lediglich einen von zahlreichen Aspekten der Objektivitätsdiskussion abzudecken, während andere stillschweigend unter den Tisch fallen. 


\subsection{Drei Argumentationsstrategien}

Ein weiterer Schritt in Richtung einer systematischen Überschau des Untersuchungsgebietes besteht darin, grundlegende Argumentationsstrategien im Umgang mit der nun eingeschränkten Fülle an Objektivitätsauffassungen zu identifizieren. Prinzipiell können drei Möglichkeiten unterschieden werden, wie mit der diversifizierten Begriffslandschaft umgegangen werden kann. Von diesen drei Argumentationsstrategien lassen sich in den einschlägigen Beiträgen zwei ausmachen, die dritte wird hier vorgeschlagen und erläutert.

(1) Die „Realdefinitionsstrategie“: Jede der Objektivitätsvarianten kann jeweils als Realdefinition von Objektivität betrachtet werden. Die Ansprüche der Vertreter der jeweiligen Begriffsvariante schließen sich gegenseitig aus. ${ }^{15}$ Meist wird dieser Anspruch nicht explizit gemacht, sondern einfach die jeweils eigene Objektivitätsauffassung den jeweiligen Überlegungen zugrundegelegt und ihre Ausschließlichkeit stillschweigend vorausgesetzt. Mit der Realdefinitionsstrategie ist ein Reduktionsanspruch verbunden, der weit über eine grobe, erste begriffliche Explikation hinausgeht (wie sie gerade eben mittels der Vorstellung einer Freiheit von subjektiven Verzerrungen gegeben worden ist). So nützlich eine solche erste Annäherung ist, so zwingend erforderlich ist ihre weitere begriffliche Ausdifferenzierung, um eben nicht bei einer Realdefinition stehen zu bleiben.

Warum aber sollte eine weitere Spezifikation und Ausdifferenzierung jedes ,monistischen' Definitionsversuchs erforderlich sein? Aus dem allgemeinen Sprachgebrauch geht hervor, dass „Objektivität“/,objektiv“ auf die unterschiedlichsten Gegenstandsbereiche angewandt wird. Eine monistische begriffliche Explikation oder Definition lässt bedeutende begriffliche Aspekte außen vor, die eine Antwort auf die Frage nach dem begrifflichen Gehalt von „Objektivität“/,objektiv“" an irgendeiner Stelle enthalten sollte. Analog dazu müssen Versuche scheitern, eine der ,Bereichsobjektivitäten“, ob nun die ontologische oder die methodologische Objektivität oder auch intersubjektive Geltung, als die Auffassung von Objektivität schlechthin

15 Hier kann Nozick 1998 exemplarisch angeführt werden. 
auszuzeichnen. In all diesen Fällen ist kaum einzusehen, wie ein Reduktionsanspruch eingelöst werden könnte, der die verschiedenen Verwendungsweisen des Objektivitätsbegriffs auf eine einzige Realdefinition reduzieren könnte. ${ }^{16}$ Im zum Teil bewusst konfrontativen Gegensatz zur Realdefinitionsstrategie wird daher in der Literatur eine „Koexis-

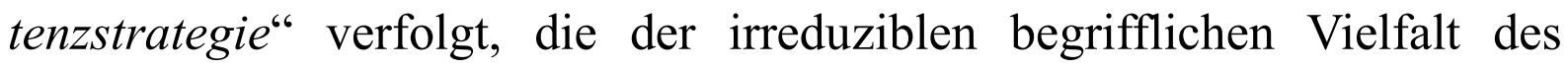
Objektivitätsbegriffs Rechnung tragen möchte.

(2) Die „Koexistenzstrategie“: Jede der begrifflichen Varianten stellt einen Versuch dar, jeweils ein Konzept von „Objektivität“/,objektiv“ zu verdeutlichen, das im Sprachgebrauch Verwendung findet. Die diversen Konzepte sind aber lediglich aus mehr oder minder kontigenten Gründen unter das Rubrum „Objektivität“ subsumiert worden, wobei die Existenzberechtigung der jeweils anderen Konzepte nicht bestritten wird. Folglich sieht dieser Ansatz eine unsystematische Koexistenz verschiedener Konzepte vor. ${ }^{17}$ Einige von ihnen stehen untereinander in bestimmten Relationen, ohne jedoch aufeinander reduzierbar zu sein. ${ }^{18}$

Die Koexistenzstrategie vermeidet zwar einen unterkomplexen Umgang mit der Begriffslandschaft, geht aber über das reine Konstatieren einer begrifflichen Vielfalt kaum hinaus. Das bloße Aufzählen und Aneinanderrei-

16 Vgl. Douglas 2004, 453. Sie unterscheidet mehrere Modi der Objektivität und plädiert für eine Irreduzibilität der einzelnen Modi. Wie sie zurecht bemerkt, führen monistische Reduktionsversuche einzig dazu, dass wichtige Elemente des Objektivitätsbegriffs unter den Tisch fallen (ebd., 467).

17 Vgl. hierfür Douglas 2004 und Megills Überblick über die Begriffslandschaft (in Megill 1994b) und auch Harding 2003, 164 f. und Patzig 1977.

18 Nach Douglas werden sie allenfalls von einer „begrifflichen Kohärenz“ zusammengehalten (Douglas 2004, 453 und 467). Vgl. auch Daston/Railton 2007: „Die meisten [...] Arbeiten über Objektivität behandeln sie als einen Begriff. [...] immer wird vorausgesetzt, Objektivität sei abstrakt, zeitlos und monolithisch. Aber wenn sie ein reiner Begriff ist, gleicht sie weniger einer Bronzeskulptur aus einem Guß als einem improvisierten, aus schlecht passenden Teilen von Fahrrädern, Weckern und Dampfrohren zusammengelöteten Apparat.“ (Ebd., 55) Tatsächlich werde unter dem Rubrum „Objektivität“ eine Vielzahl nicht zusammenhängender Bedeutungen von Objektivität zusammengestellt. „Diese unterschiedlichen Bedeutungen des Wortes, objektiv' hängen weder im Prinzip noch in der Praxis zusammen.“(Ebd., 56.) 
hen von jeweils für irreduzibel empfundenen begrifflichen Varianten wird nicht von weiteren Systematisierungsschritten gefolgt. Eine solche weitergehende Systematisierung der Begriffslandschaft ist aber möglich und auch wünschenswert, weil auf diese Weise Zusammenhänge aufgedeckt werden können, die andernfalls keine weitere Beachtung mehr erhalten würden, wodurch auch die Analyse der jeweils betrachteten Objektivitätsauffassung kompromittiert werden würde.

Den Weg, den die Koexistenzstrategie weist, indem sie darauf beharrt, die begriffliche Vielfalt der Verwendungsweisen von „Objektivität“/,objektiv“ nicht einzuebnen, möchte das hier zu entwickelnde Modell weitergehen. Die Koexistenzstrategie ist daher nicht etwa abzulehnen, wie dies für die Realdefinitionsstrategie gilt, sondern im Hinblick auf die Bedürfnisse einer Diskussion historischer Objektivität weiterzuentwickeln. Als eine solche Weiterentwicklung wird ein „Komponentenmodell“ vorgeschlagen. 


\subsection{Das Komponentenmodell}

Das Komponentenmodell als Weiterentwicklung der Koexistenzstrategie geht davon aus, dass jede der Objektivitätsauffassungen, auf welche die Untersuchung eingegrenzt worden ist, versucht, eine Komponente zu einem komplexen Gesamtbegriff beizusteuern. Die Grundidee ist hier, dass die jeweiligen begrifflichen Varianten einen unerlässlichen Beitrag zum begrifflichen Gehalt leisten, den Objektivitätsbegriff aber jeweils nicht ausschließlich ausfüllen können.

Soweit handelt es sich aber noch um keine Weiterentwicklung der Koexistenzstrategie. Doch nun treten zwei Züge hinzu: Erstens stehen die diversen begrifflichen Varianten in einem systematischen Zusammenhang, zweitens aber sind diese Elemente historischer Objektivität um einen Begriffskern gelagert, der die verschiedenen Elemente integriert.

Der Begriffskern ist korrespondenztheoretisch aufzufassen. Für den Objektivitätsbegriff ist die Vorstellung integrativ, dass sich die Darstellung ${ }^{19}$ der Vergangenheit danach zu richten hat, was geschehen ist. Darstellungen stehen in einer Korrespondenzbeziehung zum Dargestellten. Es handelt sich genauer gesagt um die Korrespondenz zwischen bestehendem Sachverhalt und einem Satzgehalt (einem propositionalem Gehalt bzw. einer Proposition). Der Satzgehalt dass $p$ korrespondiert mit dem existierenden Sachverhalt $p$; der Satzgehalt „dass der Stuhl vier Beine hat" korrespondiert mit dem Sachverhalt, dass der Stuhl vier Beine hat. Korrespondenz wird expliziert als Übereinstimmung von Satzgehalt mit bestehendem Sachverhalt.

Die Korrespondenzauffassung des Begriffskerns präzisiert die weiter oben (1.1) vorläufig und vage eingeführte absolute (epistemologische) $\mathrm{Ob}$ jektivität. ${ }^{20}$ Die Gehalte epistemisch-doxastischer Zustände (Überzeugun-

19 „Darstellung“ wird in dieser Arbeit als Gattungsbegriff für alle Arten der Präsentation historischer Erkenntnis wie etwa Interpretation, Narration, Deskription, Erklärung usf. benutzt.

20 Megill bezeichnet die korrespondenztheoretische Objektivitätsvariante als ,absolute objectivity“, ohne ihr einen besonderen Status vor den anderen von ihm vor- 
gen, Wissen, Vermutungen u. a.) sind propositionale Gehalte, die sprachlich erfassen, ,wie die Dinge liegen' oder, wie sie (an sich) sind', sprich, einen (existierenden) Sachverhalt sprachlich erfassen. Die absolute Objektivität definiert sich über die auf diese Weise explizierte Korrespondenz zwischen Satzgehalt und Sachverhalt. Absolute Objektivität liegt dann vor, wenn der sprachlich erfasste Sachverhalt besteht und gleichzeitig der ihn ausdrückende Satzgehalt mit diesem bestehenden Sachverhalt übereinstimmt.

In dieser Bestimmung von Korrespondenz stecken einige Voraussetzungen: (1) Die Übereinstimmung besteht nicht zwischen dem Geist im allgemeinen oder einem Begriff im besonderen und einem Gegenstand in der Welt, sondern zwischen der Konfiguration bestimmter Eigenschaften (intrinsischen oder relationalen) von Gegenständen, kurz: einem Sachverhalt, und einem (zugegebenermaßen ontologisch schwer fassbaren $)^{21}$ sprachabhängigen Gebilde namens „Proposition“ (oder „Satzgehalt“ bzw. ,propositionaler Gehalt"s).

(2) Zwar wird insbesondere mit dem Ausdruck „Satzgehalt" Satzförmigkeit nahegelegt, worum es aber hierbei nicht geht, ist die Übereinstimmung eines Satz-tokens oder eines Satz-types mit den Sachverhalten der Vergangenheit. Sowohl Satz-tokens, i. S. v. geäußerter Satz-type, als auch Satz-types, i. S. v. instantiierbare Satzform, sind nur insofern potentielle Korrespondenz-Träger, als sie Propositionen ausdrücken. ${ }^{22}$ Unser Sprachgebrauch weist darauf hin. Wir sprechen ständig davon, dass wir mit unterschiedlichen Sätzen dasselbe aussagen oder denselben Gedanken ausdrücken oder dasselbe meinen, was bedeutet, dass wir intuitiv von einem

gestellten Objektivitätsvarianten zuzuerkennen (ders. 1994b, 1 und 2-5). Auch Patzig 1977 hat eine Tendenz, eine korrespondenztheoretische Objektivitätsauffassung als die zentrale anzunehmen, ohne dies jedoch explizit zu machen. Ebenso scheint Acham 1977 implizit der korrespondenztheoretischen Objektivität (,,repräsentative Gegenstandserfassung“) einen Vorrang einzuräumen.

21 Horwich 1994b, xii: ,[..] an accurate account of truth - one faithful to our actual conception of it - would seem not to permit a sceptical attitude towards propositions, but rather to call for a greater effort to understand them."

22 Vgl. Horwich 1994b und Kirkham 1992 für ein Plädoyer für eine liberale Einstellung gegenüber potentiellen Korrespondenzrelata bzw. Wahrheitsträgern. 
ausdrucksunabhängigen, wenngleich nur sprachlich zugänglichen Gehalt, eben einem propositionalen Gehalt, ausgehen, der dann auf seine Übereinstimmung mit den von ihm zum Ausdruck gebrachten Tatsachen ausdrucksunabhängig beurteilt werden kann. ${ }^{23}$ Hinter den jeweils ausdrückenden Satz-tokens und den von ihnen instantiierten Satz-types stehen andere Entitäten, eben Propositionen, die von ihnen ausgedrückt werden. ${ }^{24}$ Trotz der Vorrangigkeit von Propositionen soll im folgenden auch von der Korrespondenz von Aussagen gesprochen werden. Wenn Propositionen auch die primären Kandidaten für die Rolle des Korrespondenz-Relatums sind, so finden sie doch im wissenschaftlichen Kontext ihren Ausdruck in Aussagen.

(3) Von Korrespondenz kann nur dann gesprochen werden, wenn der ausgedrückte Sachverhalt auch besteht. Eine Proposition bzw. die sie ausdrückende Aussage korrespondiert nur dann, wenn das, was sie ausdrückt, auch existiert, also eine Tatsache ist. Besteht der Sachverhalt, der behauptet wird, nicht, kann keine Korrespondenz bestehen. Falschheit besteht demnach darin, dass die Korrespondenz scheitert, weil der behauptete Sachverhalt in der Form, in der er behauptet wird, so nicht besteht. ${ }^{25}$

Was aber kann genauer unter Korrespondenz verstanden werden? Dass es sich dabei um eine Übereinstimmungsrelation zwischen den beiden Relata „bestehender Sachverhalt“ und „Satzgehalt“" handelt, ist zunächst einmal nicht besonders informativ. Die Antwort kann jedenfalls nicht in einer Strukturübertragung von einer Konstellation von Gegenständen und ihren

23 Allein die Möglichkeit, denselben Sachverhalt in verschiedenen Sprachen mit z. T. gravierend differierender Ausdrucksstruktur ausdrücken zu können, dürfte als ausreichender Beleg gelten.

24 Wir besitzen außerdem ein starkes Indiz für die Existenz von Propositionen (oder etwas ihnen vergleichbares) durch die Analyse von sog. ,,propositionalen Einstellungen“" in einen intentionalen ,Modus', sc. den des Meinen, Bezweifelns, Glaubens etc., und einen propositionalen Gehalt, also einen satzförmigen ,Gegenstand', der gemeint, bezweifelt oder geglaubt werden kann. Der propositionale Gegenstand kann unabhängig vom jeweiligen Ausdruck von Modus zu Modus und von Person zu Person, weitergegeben ' werden.

25 Vgl. Austin 1994, 179, Anm. 23: „To be false is (not, of course, to correspond to an non-fact) to miscorrespond with a fact.“ 
Eigenschaften auf die Proposition oder eine Aussage (wie dies Wittgenstein im Tractatus zu entwickeln suchte) ${ }^{26}$ bestehen. Ein solcher Isomorphismus scheitert aus einer ganzen Reihe von guten Gründen. ${ }^{27}$ Die Antwort muss darin gesucht werden, dass wir, wenn wir eine Proposition mitgeteilt bekommen (sei es in Form einer Aussage oder eines propositionalen Gehalts einer propositionalen Einstellung ${ }^{28}$ ), wir intuitiv wissen, wie die Welt beschaffen sein muss bzw. müsste, damit die Proposition mit dem Sachverhalt übereinstimmt. Das kann auch bedeuten, den Sachverhalt erst noch in die Welt zu bringen (etwa wenn der propositionale Gehalt Teil eines Imperativs oder eines Wunsches ist). Nichtsdestotrotz müssen wir auch in diesem Fall wissen, wie die Welt beschaffen sein muss, damit eine Übereinstimmung zwischen Proposition und Sachverhalt bestehen kann.

Voraussetzung für diese intuitive Fähigkeit ist Sprachkenntnis, das heißt Kenntnis von der Bedeutung und Verwendung der Begriffe einer Sprache. Besitzen wir diese Kompetenz - und Teil unserer Sozialisation besteht im Erwerb dieser Sprachkompetenz - und haben wir das nötige empirische Wissen, dann sind wir dazu in der Lage, intuitiv zu entscheiden, ob eine Korrespondenz zwischen einem Satzgehalt und einem von ihm ausgedrückten Sachverhalt besteht oder nicht.

An dieser Stelle ist eine Ausweitung des Anwendungsbereichs von Objektivität im Korrespondenzsinn notwendig. Die philosophische Diskussion von „Korrespondenz“ findet auf der Ebene von einzelnen Aussagen oder Propositionen statt. Zum Zweck der explizierenden Einführung der Begriffe ist dies ausreichend. Demgegenüber beschäftigt sich aber die Geschichtswissenschaft in der Regel nicht so sehr mit der Objektivität einzel-

26 TLP $2.1 \mathrm{ff}$.

27 Vgl. dazu Newman 2002 und Kirkham 1992, chap. 4. Um nur stichwortartig einige Schwierigkeiten zu nennen: Wie sieht es mit negativen Tatsachen aus? Welche Strukturen werden in komplexen Aussagen übertragen? Wie sieht die Strukturübertragung bei Implikationen aus?

28 Der propositionale Gehalt, dass die Tür geschlossen ist, kann in den verschiedensten Kontexten auftauchen: in propositionalen Einstellungen wie der Überzeugung, dass die Tür geschlossen ist; in Sprechakten wie Imperativen: „Schließe die Tür!'‘; in optativischen Einstellungen wie dem Wunsch, dass die Tür geschlossen sein möge etc. 
ner isolierter Aussagen, sondern mit der größerer Aussagenkomplexe, weil sie, wofür später noch argumentiert werden wird, wesentlich narrativen Charakters ist - in den Worten A. Dantos: „History tells stories“ ${ }^{\text {(29. }}$. Für die Geschichtswissenschaft ist aus diesem Grund eine Ausweitung unerlässlich.

Die Objektivität, und das heißt nach dem hier eingeführten Modell: die Korrespondenz dieser Darstellungen und ihrer Teile kann aber nicht ohne weiteres von der Ebene einzelner Aussagen auf die Ebene größerer Aussagenkomplexe übertragen werden. Dass diese Übertragung aber stattfinden muss, zeigt allein schon die unproblematische, alltägliche Verwendung von Ausdrücken wie ,zutreffen“, „korrekt sein“ oder „objektiv sein“, die ständig auf Darstellungen aller Art, wie Protokolle, Aufsätze, Erzählungen, Anekdoten, Berichte o. ä., aber auch und vor allem auf historische Darstellungen angewandt werden.

Ein Mittel, um die Übertragung von der Ebene einzelner Propositionen oder Aussagen auf die Ebene zusammenhängender Aussagenkomplexe zu leisten, sind die wahrheitsfunktionalen Operatoren der Aussagenlogik, und das bedeutet hier in erster Linie, die Konjunktion. Mit ihr könnten die Wahrheitswerte der einzelnen objektiven Aussagen erhalten und zu einem Wahrheitswert der gesamten historischen Darstellung, aufaddiert' werden. Dass aber historische Darstellungen keine Konjunktionen von Aussagen sind, die in dem Augenblick falsch werden, in dem auch nur eine einzige falsche Aussage darin enthalten ist, muss wohl kaum auseinandergesetzt werden. Jeder, der einen solchen Vorschlag macht, befindet sich sofort in einer unhaltbaren Position, nicht nur weil eine einzige falsche Aussage die gesamte Darstellung falsch werden ließe, sondern auch weil jede historische Darstellung Aussagen enthalten wird, die keinen festgelegte Wahrheitswert besitzen (etwa wenn begriffliche Mehrdeutigkeiten vorliegen) oder weil für die Geschichtswissenschaft essentielle Zusammenhänge aussagenlogisch nicht erfassbar sind (insbesondere an die kausale Begründung durch Weil-Sätze ist dabei zu denken).

Daher wird in der Literatur zwischen der Wahrheit einzelner Aussagen und der Angemessenheit (i. e. Objektivität) historischer Darstellungen un-

29 Danto 1980, 184. 
terschieden. ${ }^{30}$ Diese Unterscheidung ist nicht besonders glücklich, weil gerade der alltägliche Sprachgebrauch auch größeren Aussagekomplexen dann Wahrheit (neben den anderen angeführten Qualifikationen) zuschreibt, wenn die Darstellung grosso modo den dargestellten Sachverhalten korrespondiert, ohne damit aber zu meinen, dass jede Aussage wahr und die gesamte Darstellung eine Konjunktion von wahren Aussagen ist. Sinnvoller ist es, diesen Sprachgebrauch nicht künstlich einzuengen, sondern darauf zu verweisen, dass wir als Leser historischer Darstellungen dazu in der Lage sind, bestimmte Darstellungen oder Teile davon als korrespondierend oder nicht-korrespondierend zu bezeichnen, ohne dass wir dabei eine gewaltige Konjunktion vor Augen hätten, mit deren Hilfe wir aus den Wahrheitswerten der Einzelaussagen den Wahrheitswert der Darstellung, errechneten'. Vielmehr ist es, wie gesagt, möglich, intuitiv einzuschätzen, ob die Darstellung korrespondiert oder nicht. Wir müssen über das entsprechende sachlich-empirische, in erster Linie aber sprachlich-begriffliche Wissen verfügen, um dies tun zu können.

Dass das Komponentenmodell historischer Objektivität in dem Augenblick, in dem es die Korrespondenz in den Kern des Modells rückt, in dieselbe Notlage wie die Korrespondenztheorie der Wahrheit kommt, erklären zu müssen, was Korrespondenz ist, ist unvermeidlich. Aber ebenso wenig wie die Korrespondenztheorie der Wahrheit als intuitiv plausibelste und auch insofern aussagekräftige Wahrheitsexplikation, als kompetente Sprecher wissen, was damit gemeint ist, wenn sie es auch nicht mit theoretischer Sicherheit festhalten können, bislang von konkurrierenden Wahrheitsdefinitionen zufriedenstellend abgelöst werden konnte, ${ }^{31}$ muss hier auf die Korrespondenz als Begriffskern verzichtet werden, nur weil es notorisch schwierig ist, eine theoretisch adäquate, exakte Definition von Korrespondenz zu geben. Unser intuitives Verständnis davon, wann Korrespondenz vorliegt und wann nicht, muss genügen, weil aus dem Vorhandensein der Fähigkeit zum Verständnis und zur Anwendung dieses theoretisch un-

30 Vgl. z. B. Ankersmit 1983, Gorman 1998, Lorenz 1998 b.

31 Vgl. dazu Wrights Argumentation, dass jede zufriedenstellende Explikation des Wahrheitsbegriffs notwendig eine Form der Korrespondenz beinhalten sollte (ders. 2001 und 2003); vgl. auch Horwich 1994b, xii f. und Kirkham 1992, chap. 4. 
klaren Konzepts immerhin geschlossen werden kann, dass es sich nicht um einen leeren oder unbrauchbaren Begriff handelt.

Dass es sich bei der korrespondenztheoretischen Explikation des Begriffskerns von Objektivität um die klassische korrespondenztheoretische Explikation des Wahrheitsbegriffs handelt ${ }^{32}$, macht einige Bemerkungen zur Abgrenzung von Wahrheit und Objektivität notwendig. ${ }^{33}$ Inwiefern unterscheidet sich der korrespondenztheoretisch explizierte Begriffskern (i. e. absolute Objektivität) und damit das Komponentenmodell vom korrespondenztheoretischen Wahrheitsbegriff? Ist mit Objektivität nicht im Grunde die Wahrheit der Darstellung gemeint?

Im Anspruch, objektive Erkenntnisse vorgelegt zu haben, steckt implizit der Anspruch, bestimmte (methodische) Standards auf dem Weg der Erkenntnisgewinnung eingehalten $\mathrm{zu}$ haben und nicht etwa geraten oder sich auf die Stimme einer Autorität verlassen zu haben. Wer also von objektiver historischer Erkenntnis bzw. objektiven historischen Darstellungen spricht, der meint damit nicht nur, dass die Darstellung mit der Vergangenheit korrespondiert, sondern auch, dass diese Darstellung auf bestimmte Weise erarbeitet worden ist. Der Historiker hat sich als unvoreingenommener Historiker gezeigt, bestimmte Methoden verwandt und beides ist ihm zusammen

32 Vgl. dazu Tugendhat 1979, Tugendhat/Wolf 1983, Newman 2002, Seebaß 2011, 23-33 (,der rigide Wahrheitsbegriff") sowie die klassische Passage in Aristoteles Metaphysik IV,7 (1011b, 26 ff.): „Zu sagen nämlich, das Seiende sei nicht oder das Nicht-Seiende sei, ist falsch, dagegen zu sagen, das Seiende sei und das Nicht-Seiende sei nicht, ist wahr." Vgl. auch die ,modernen Klassiker' Russell 1994 und Austin 1994.

33 Vgl. etwa Gormans Untersuchung über das Verhältnis von Wahrheit und Objektivität, die damit beginnt, dass die eigentliche Bedeutung von Objektivität die Objektivität von Aussagen ist, die sich nicht davon unterscheide, was man eigentlich als Wahrheit bezeichne. „This concept of ,objective“ is [..] to be used fundamentally as a predicate of statements; as such, ,objective" means little more than ,true“." $(1998,320)$ Gorman hält dies für falsch. Es lässt sich beobachten, dass in einer Reihe von Veröffentlichungen Wahrheit und Objektivität immer wieder vermischt werden. Als Beispiele mögen hinreichen: Appleby et al. 1994, Novick 1988, McCullagh 1998 und 2004a, Pompa 1991, 114 f. Vgl. Agazzi 1988, der glaubt, Objektivität hätte Wahrheit als zentrale Kategorie der Wissenschaftlichkeit ersetzt: „[L]'objectivité a remplacé l'idée de vérité.“ (17 f.) 
mit der Güte seiner Ergebnisse vom fachdisziplinären Konsens im Großen und Ganzen bestätigt worden. Es schlägt sich im Objektivitätsbegriff also die Art der Erkenntnisgewinnung nieder. Um für objektiv gelten zu können, ist es für eine Darstellung nicht ausreichend, mit den dargestellten Tatsachen übereinzustimmen, ebenso wichtig ist, wie sie erlangt worden ist. Die Wahrheit einer Aussage und einer Darstellung dagegen ist von der Art der Erkenntnisgewinnung unabhängig. Wahre Aussagen können durch welches Manöver auch immer erreicht werden. Man kann raten, hellsehen, wissenschaftlich vorgehen oder durch Zufall über eine Erkenntnis stolpern. Objektivität dagegen impliziert, dass bestimmte Erkenntnisse auf eine bestimmte Art und Weise gewonnen worden sind. Im Objektivitätsbegriff konvergiert der Aspekt der Erkenntnisgewinnung mit dem Aspekt der Korrespondenz der Darstellung.

Ein immer wieder vorgeschlagenes weiteres Charakteristikum der Objektivität, das sie vom Wahrheitsbegriff unterscheiden soll, ist ihre Graduierbarkeit. ${ }^{34}$ Zentral scheint hier zu sein, dass eine Darstellung objektiver als eine andere sein kann - auch wenn beide „den Tatsachen entsprechen“ -, sobald sie mehrere Perspektiven ,integriert ${ }^{\text {' }}{ }^{35}$ Eine eingehende Explikation dessen, was damit gemeint ist, steht im Augenblick noch aus. Vorläufig kann damit die folgende Reihe von Grundgedanken identifiziert werden, die meist ineinander übergehen.

Die meisten historischen Darstellungen integrieren bereits durchgeführte

34 Vgl. Bevir 1994, S. 337, Douglas 2004, Haskell 2004, ders. 1998, Nagel 1986.

35 Die historische Perspektivenintegration darf nicht mit Nagels Vorstellung eines Kontinuums immer objektiverer Beschreibungen der Welt verwechselt werden. Nagel geht es um die Frage, inwieweit eine individuell-subjektive Perspektive immer weiter bis hin zu einem enthumanisierten View from nowhere transzendiert werden kann (1991, 116 f.). Für die Geschichtstheorie ist jedoch weder die rein subjektive Perspektive, wie sie etwa durch die phänomenale Wahrnehmung des jeweiligen Erkenntnissubjekts exemplifiziert wird, noch die alle menschlichen Perspektiven transzendierende Perspektive eines, idealen' Physikalismus sonderlich interessant. An der grundsätzlichen Bedeutung von Nagels Erkenntnis, dass es nicht eine strenge Dichotomie zwischen subjektiv und objektiv gibt, sondern ein Kontinuum objektiverer Perspektiven, ändert sich dadurch nichts, auch wenn diese Erkenntnis an die spezifische Situation der Geschichtstheorie assimiliert werden muss. 
Untersuchungen und damit die Perspektiven, unter denen diese Untersuchungen durchgeführt worden sind, in die eigene Darstellung. Damit geht meist eine (von dieser ersten Form der Perspektivenintegration zu unterscheidende) Form einher, die als Wechsel der Betrachtungsebene bezeichnet werden könnte. Die Perspektive der Alltagsgeschichte könnte von der Perspektive der Strukturgeschichte integriert werden. ${ }^{36}$ Die erste theoretische Zugangsweise widmet sich einzelnen, alltäglichen Lebensformen und individuellen Schicksalen, während die zweite grundlegende Strukturen im Verlauf der Geschichte entdecken möchte. Letztere könnte auf einer , höheren Ebene' die erste integrieren.

Eine historische Darstellung kann auch für objektiver als eine andere gehalten werden, wenn es ihr gelingt, umfangreicher zu sein als eine andere Darstellung. „Umfangreich“ kann bedeuten, dass mehr erklärungsbedürftige Phänomene erklärt werden als in anderen Arbeiten. Es kann aber auch bedeuten, dass mehr Sachverhalte in die Darstellung integriert werden als in einer konkurrierenden Darstellung (wobei hier das Problem entsteht, dass Sachverhalte sinnvoll zählbar sein müssen). Oder aber es kann bedeuten, dass weniger nicht-korrespondierende Aussagen in einer Darstellung enthalten sind als in einer anderen.

In der Regel werden alle diese Momente, und vielleicht auch einige weitere, gemeinsam eine Darstellung vor einer anderen als objektiver auszeichnen. Wahrheit muss dagegen von einer strengen Dichotomie ausgehen. Entweder ist eine Darstellung wahr oder sie ist es nicht.

Doch warum sollte der korrespondenztheoretische Begriffskern ein unerlässlicher Bestandteil eines Komponentenmodells sein? Wer darauf verzichtet, die Korrespondenz historischer Darstellungen in sein Objektivitätsmodell aufzunehmen und sich stattdessen darauf beschränkt, historische Objektivität ausschließlich über andere Objektivitätsauffassungen begrifflich zu explizieren, wird feststellen, dass er damit auf Objektivität als solche verzichtet hat. Denn selbst bei aller Umsicht und wissenschaftlichen Kompetenz kann kein Historiker ausschließen, dass er nicht-korrespondierende Ergebnisse zutage gefördert hat, obwohl er sämtlichen wissenschaft-

36 An dieser Stelle muss man von einigen sich gegenseitig exkludierenden Überzeugungen der beiden Richtungen absehen (vgl. Raphael 2003). 
lichen Standards gerecht geworden ist. Nicht-korrespondierende Forschungsergebnisse und historische Darstellungen können jedoch schlechterdings nicht als objektiv bezeichnet werden. Zumindest also muss der Anspruch, dass historische Erkenntnisse bzw. Darstellungen mit der Vergangenheit korrespondieren, Teil eines Objektivitätsmodells sein, wenn auch klar ist, dass das tatsächliche Vorliegen eines Korrespondenzverhältnisses nicht unumstößlich ein für allemal festgestellt werden kann.

Unerlässlich für das Modell sind aber auch die übrigen Komponenten. Denn erstens hat die Abgrenzung des Objektivitätsbegriffs vom Wahrheitsbegriff erbracht, dass Wahrheit, nicht aber Objektivität darauf verzichten kann, Aufschlüsse über den Weg der Erkenntnisgewinnung und die Einhaltung gewisser epistemologischer Standards zu geben. Die übrigen drei Objektivitätsauffassungen spielen also insofern eine unerlässliche Rolle, als eine bloße Korrespondenzbeziehung zwischen Darstellung und Dargestelltem nicht ausreicht, um wirklich von Objektivität sprechen zu können. Zweitens - das wurde eingangs im Zusammenhang mit der Realdefinitionsstrategie bereits angeführt - ist unser Sprachgebrauch so eingestellt, dass wir mehrere Objektivitätsauffassungen nebeneinander verwenden, ohne eine Notwendigkeit zur begrifflichen Reduktion zu sehen. Die alternativen Begriffsvarianten sind in diesem Modell also nicht nur eine Dreingabe oder bloßes Dekorum. Aus diesem Grund genügt es nicht, im Sinne der Realdefinitionsstrategie, den korrespondenztheoretischen Objektivitätsbegriff als den Objektivitätsbegriff schlechthin auszuzeichnen.

Doch die Unerlässlichkeit jeder der Komponenten verbürgt noch kein Komponentenmodell. Zwar schließt die Unerlässlichkeit der einzelnen Objektivitätsauffassungen eine monistische Reduktion im Sinne einer Realdefinitionsstrategie aus, doch damit allein wäre das Komponentenmodell noch keine Weiterentwicklung der Koexistenzstrategie, die auch eine bloß unverbundene Aufzählung irreduzibler Objektivitätsauffassungen erlauben kann. Vielmehr muss das Komponentenmodell zugrundegelegt werden, dessen Kern vom korrespondenztheoretischen Objektivitätsbegriff gebildet wird, und dessen weitere Komponenten jedoch eine Reihe von anderen unerlässlichen Objektivitätkonzeptionen sind, die in einem besonderen Verhältnis zum Begriffskern stehen. Damit sollen einerseits die verschiedenen 
irreduziblen Aspekte des Objektivitätsbegriffs erhalten bleiben, andererseits aber auch eine bloße Koexistenz vermieden werden.

Um eine bloße Koexistenz zu vermeiden, sollen die Beziehungen der übrigen drei Objektivitätsauffassungen, die nach der Eingrenzung des Untersuchungsbereichs verblieben sind - also Objektivität als Unparteilichkeit, methodologische und konsensuale Objektivität -, zum Begriffskern erörtert werden. Zum Begriffskern stehen sie in einer besonderen, einer derivaten Beziehung, das heißt, die übrigen Objektivitätsauffassungen sind für sich betrachtet nur derivative Begriffsvarianten, die nicht vollständig erfassen, was Objektivität ist, dabei aber vom korrespondenztheoretisch explizierten Begriffskern zu einem Gesamtkonzept integriert werden. 


\subsection{Die derivativen Objektivitätskomponenten}

„Derivativ“ impliziert eine Abhängigkeitsrelation. Welche Abhängigkeit besteht zwischen Begriffskern und den derivativen Komponenten? Den drei derivativen Objektivitätsauffassungen ist gemeinsam, dass sie in einem Mittelverhältnis zum Begriffskern stehen. Als Mittel sind sie zwar ebenso unerlässlich wie der Begriffskern, wenn es darum geht, objektive Darstellungen $\mathrm{zu}$ erhalten und $\mathrm{zu}$ rechtfertigen, sie sind jedoch für das „produktive Potential“ der Geschichtswissenschaft nutzlos, wenn die Ergebnisse der Forschung nicht mit der Vergangenheit korrespondieren - umgekehrt gilt dies jedoch nicht. Mit der Vergangenheit lediglich korrespondierende Darstellungen sind zwar nicht als objektiv zu bezeichnen, können aber, als Darstellungen, die faktisch mit der Vergangenheit korrespondieren, immer noch ihren Nutzen haben. So unerlässlich für eine gelungene begriffliche Explikation historischer Objektivität alle vier Komponenten auch sein mögen, so nutzlos und auf ihre Rolle als Mittel zu einem Zweck reduziert sind die derivativen Objektivitätsauffassungen ohne den Begriffskern. In ihrem Mittelcharakter und in ihrer Nutzlosigkeit für die Geschichtsschreibung können die methodologische und die konsensuale $\mathrm{Ob}$ jektivität wie auch die Objektivität als Unparteilichkeit als derivativ bezeichnet werden.

In den folgenden drei Abschnitten (1.4.1-1.4.3) soll für die drei derivativen Komponenten kurz nachgewiesen werden, dass sie tatsächlich in einem Mittelverhältnis zum Begriffskern stehen. Gleichzeitig muss gezeigt werden, ob der Anspruch des Komponentenmodells, eine Weiterentwicklung der Koexistenzstrategie zu sein, sich auch auf Beziehungen unter den derivativen Objektivitätsauffassungen stützen kann. Das soll geschehen, indem neben der Abhängigkeitsbeziehung der derivativen Komponenten zum Begriffskern einige Ordnungsbeziehungen unter den derivativen Komponenten angedeutet werden.

Folglich ergibt sich für die folgenden drei Abschnitte ein doppeltes Argumentationsziel: Für jede der derivativen Objektivitätsauffassungen wird erstens nachgewiesen werden, dass sie in einem Abhängigkeitsverhältnis 
zum Begriffskern stehen, und zweitens werden sie untereinander in ein Ordnungsverhältnis gebracht.

\subsubsection{Methodologische Objektivität}

Zunächst soll die methodologische oder prozedurale Objektivität untersucht werden. ${ }^{37}$ Sie beinhaltet die Grundvorstellung, Verzerrungen auszuschalten, indem der individuellen Urteilskraft und damit der individuellen Willkür durch objektive Verfahrensanweisungen und methodologische Standards Grenzen auferlegt werden.

In der allgemein wissenschaftstheoretischen Literatur zum Thema Objektivität werden meist für diese Form der Objektivität die Versuche, qualitative Methoden durch quantitativ-statistische Methoden zu ersetzen, ${ }^{38}$ herangezogen. Doch für die Geschichtswissenschaft spielt diese Form der prozeduralen Objektivität keine zentrale Rolle, obwohl durchaus mit Erfolg versucht wird, quantitativ-statistischen Methoden eine prominente Stelle einzurichten. Der Grundgedanke prozeduraler Objektivität tritt hier aber besonders deutlich hervor. Die Rolle des Subjekts soll darauf beschränkt werden, einen eindeutig und präzise definierten Prozess zu initiieren, dessen Ergebnisse nicht mehr vom Subjekt, sondern den (zuvor definierten) Prozessparametern ,kalkuliert‘ werden. Die Gelegenheitsfenster, die sich für eine Einmischung und damit eine Verzerrung der Ergebnisse öffnen, sollen auf diese Weise minimiert, wo nicht auf Null zurückgefahren werden. Dieses Ziel kann, nach diesem Ansatz, am besten dadurch erreicht werden, dass interpretationsbedürftige qualitative Termini durch (vermeint-

37 Den Terminus ,prozedurale Objektivität“ übernehme ich von Megill 1994b, 1 und $10 \mathrm{f},,[\ldots]$ its governing metaphor is tactile, in the negative sense of ,hands off! ‘ Its motto might well be ,untouched by human hands'." (Ebd., 10) Vgl. Daston 1998, 34 und Novick 1988, 52. Letzterer ist gar der Überzeugung, dass es sich bei der „transpersonal replicability“, seinem Terminus für prozedurale Objektivität, um die „perhaps most coherent definition of objectivity“ handle.

38 Vgl. Porter 1994, 197-206. 
lich) eindeutig definierte und (vermeintlich) kontextunabhängig anwendbare Termini ersetzt werden, was am besten durch Termini erreicht werden kann, die durch statistische Größen oder überhaupt numerische Werte definierbar sind, mit deren Hilfe dann bislang nur qualitativ beschriebene Sachverhalte eine statistisch-numerische Behandlung erfahren können.

Für die Geschichte ist jedoch die Methode der Quellenkritik von größerer Bedeutung, weil sie gemeinhin als die für die Geschichtswissenschaft essentielle und auch objektivitätsverbürgende Methode angesehen wird. Es dürfte daher kaum überraschen, dass diese Untervariante der prozeduralen Objektivität bereits recht früh Anspruch darauf erhoben hat, mit historischer Objektivität identisch zu sein. Typischerweise heißt es, historiographische Interpretationen müssten durch das „Säurebad“ der Quellenkritik gehen, um als wahr gelten zu können. Mit dieser Methode wird i. d. R. Ranke $^{39}$ in Verbindung gebracht bzw. die Schule des Historismus als Pflanzstätte dieser Methodik betrachtet. ${ }^{40}$ Die Bedeutung der Quellenkritik bedarf für die Geschichtswissenschaft nicht eigens der Betonung. Die Frage ist aber, ob damit die ,neutrale' Methode gefunden worden ist, die eine

39 Mit Bezug auf die „Rankean vision of historical objectivity“, die hier unter der prozeduralen Objektivität rubriziert wird, resümiert Rüsen: „The objectivity of the research guaranteed the objectivity of the researcher." (Rüsen 2000, 64) Damit ist in nuce der Sinn und Zweck der prozeduralen Objektivität erfasst. Vgl. auch Porter: „To diminish the domain of personal judgement does not require anything so wonderful as untainted truth. It is enough that there be rules, perhaps quite conventional ones, that limit the exercise of discretion." (Ders. 1994, 197)

40 Zur Vorreiterrolle, die die deutsche Geschichtswissenschaft mit der Fokussierung auf die Methode der Quellenkritik und der Betonung „objektiver“ Wissenschaft international (und vor allem für die US-amerikanische Historiographie) gespielt hat, vgl. Appleby et al. 1994, $72-76$ und passim. Vgl. auch Beards (in 1964) Hinweis auf die Bedeutung der von Ranke begründeten Methodik. Kritisch äußert sich Iggers zum vermeintlichen Positivismus Rankes (1997, 86-88). Für Iggers hat sich gerade die US-amerikanische Rezeption Rankes in den 1880er Jahren auf ein völlig falsches Bild von Ranke versteift, das den politisch engagierten und theoretisch-philosophisch interessierten Wesenszug an Ranke völlig außer acht gelassen habe. Die damals prominenten US-amerikanischen Propagandisten des reinen (,historistischen") Faktensammeln könnten sich deshalb, so Iggers, nicht wirklich auf Ranke berufen. 
vorurteilslose Durchforstung der Quellen ermöglicht, ob also ein Objektivitätsbegriff gefunden worden ist, der für sich selbst bestehen kann, also nicht derivativ ist.

Damit Quellenkritik (als Variante prozeduraler Objektivität) als eine nicht-derivative Objektivitätsvariante gelten kann, müsste sie ein ebenso mechanisches wie Verzerrungsfreiheit garantierendes Verfahren sein. Das ist sie jedoch nicht, denn die Vorstellung, man könne gewissermaßen voraussetzungslos Fakten aus den Quellen heraussieben, ist unhaltbar. Wir besitzen keine sprachunabhängige Zugangsweise zu Fakten, das heißt, wir besitzen kein ,Wahrnehmungsorgan', das es uns erlaubt, Fakten zu ,sehen“, ohne sie begrifflich zu erfassen. Fakten sind nur in Aussagen (Propositionen) ausdrückbar. Darin liegt eine fundamentale Abhängigkeit der Quellenkritik vom jeweiligen begrifflich-theoretischen Instrumentarium des Historikers begründet, und damit ist eine quasi-mechanisch, methodischprozedural nicht zu beseitigende ,Verzerrung' in der Welt. Daneben spricht die Quelle nicht aus sich heraus zum Historiker, sondern bedarf seines selektiven Interesses, um überhaupt erst Fakten preisgeben zu können.

Diese und andere den reinen Prozesscharakter streng limitierende Elemente bei der Deutung von Quellen haben zur Folge, dass Quellenkritik nur in einem sehr abstrakten Sinn als Methode bezeichnet werden kann, keinesfalls aber als eine genau umrissene Standardprozedur mittels derer um die Welt verteilte Geschichtsforscher zu eindeutigen, gleichartigen und strikt reproduzierbaren Ergebnissen kommen (wie etwa ein Physiker, der die experimentelle Anordnung eines Kollegen nachstellt), mithin der individuelle Faktor ausgeschaltet werden kann. ,Fakten' mittels einer Verzerrungen neutralisierenden Methode oder Prozedur gleichsam mechanisch an den Tag zu fördern, ist daher ausgeschlossen. Das Ziel der Quellenkritik, objektive Fakten durch eine neutrale Methode beibringen zu wollen, kann folglich so nicht verwirklicht werden.

Zwar mag die prozedurale Objektivität in Form der Quellenkritik als ein methodologisches, aber sehr abstraktes Ideal für die Entwicklung der Geschichtsschreibung zur Geschichtswissenschaft tatsächlich von großer Bedeutung gewesen sein, unübersehbar bleibt die derivative Rolle dieser $\mathrm{Ob}$ jektivitätsform. Denn neben dem Scheitern ihres Anspruchs darauf, die 
richtige Auffassung von Objektivität zu sein, steht sie aufgrund ihrer $A b$ zweckung, sc. ein Korrespondenzverhältnis zwischen Darstellung und Dargestelltem erreichen zu wollen, in einem Abhängigkeitsverhältnis zum korrespondenztheoretisch explizierten Begriffskern. Der verfolgte Zweck - ein Ergebnis zu erreichen, das unverzerrt von subjektiver Willkür ist und damit den Tatsachen, nicht aber den Wünschen und Vorstellungen des Subjekts korrespondiert - weist darauf schon zur Genüge hin. Man könnte sogar so weit gehen zu sagen, dass die prozedurale Objektivität alles in allem nichts anderes versucht, als eine lange Tradition methodologischer Überlegungen (in der Philosophie wie auch in den Einzelwissenschaften) zu einer alles umfassenden Objektivitätsvariante umzudeuten. Versuche, Methoden zu finden, um objektive Erkenntnisse zu entwickeln und ausweisen zu können, haben nicht erst mit Quantifikation, Vereinheitlichung von Maßeinheiten und von experimentellen Apparaturen oder der Durchsetzung von Verfahrensstandards (für Experimente oder Umfragen) oder mit der Besinnung auf möglichst strenge methodische Quellenabsicherung möglichst jeder Aussage begonnen, sondern sie sind ein Element unter vielen einer traditionsreichen, übergeordneten methodologischen Anstrengung, welche die Korrespondenz der Ergebnisse und Darstellungen zum Ziel hat. Diese Methoden reihen sich damit in eine Linie altbewährter anderer Methoden ein, die in gleichem Maße von sich behauptet haben, die objektivitätsverzerrende Willkür des Erkenntnissubjekts ausgeschaltet zu haben.

Prozedurale Objektivität steht aber auch neben ihrem derivativen Verhältnis zum Begriffskern zur konsensual-fachdisziplinären Objektivität in einem weiteren Abhängigkeitsverhältnis. ${ }^{41}$ Der Grundgedanke der prozeduralen Objektivität, durch ein quasi-mechanisch anwendbares Verfahren die verzerrende Willkür des Erkenntnissubjekts einzuschränken, führt auf di-

41 Vgl. Novicks Kommentar zur prozeduralen Objektivität als Definition von Objektivität: ,[I]t is objectively true that I am 5'11” tall because (and only because) all investigators agree that the technique of measuring height is to use a standardized yardstick, and anyone applying that yardstick to me will get the same result. By this criterion objectivity is a social phenomenon brought into existence by the establishment of methodological consensus. To the extent that it is professionalization which regularizes, promulgates, and enforces this consensus, objectivity cannot be said to exist before professionalization.“ (Ders. 1988, 52) 
rektem Weg in die Abhängigkeit von konsensualer Objektivität, weil individuelle Einschätzungen, Unzulänglichkeiten, Hintergrundwissen und Parteilichkeiten faktisch immer ein Problem bleiben werden. Die einzige Möglichkeit, diese individuellen Unzulänglichkeiten und Einschränkungen der prozeduralen Objektivität wenigstens auf ein Mindestmaß zu beschränken, führt über die Kontrolle und Sanktionierung durch die scientific community. Denn (1) auch die ,objektivsten“ Regeln, Verfahrensanweisungen etc. bedürfen der Festlegung durch eine Gruppe. (2) Die Anwendung dieser Regeln etc. auf neue oder unklare Fälle ist der individuellen Einschätzung überlassen oder muss wiederum durch (konventionelle) Anweisungen geregelt werden. ${ }^{42}$ Zuletzt (3) können Regeln dazu führen, dass bestimmte, eigentlich relevante Sachverhalte nicht berücksichtigt werden, weil sie nicht regelkonform erfasst werden können oder dürfen, ${ }^{43}$ was wiederum von der neuerlichen Besinnung der Wissenschaftsgemeinschaft und einem daran anschließenden neuerlichen Konsens ausgeglichen werden muss. Hier ist gerade bei der Bearbeitung historischer Quellen der Interpretationsspielraum enorm groß und stark vom Konsens der scientific community abhängig.

Darüber hinaus steht prozedurale Objektivität in einem weiteren Abhängigkeitsverhältnis zur Objektivität als Unparteilichkeit, weil der vorgängige Zweck prozeduraler Objektivität darin besteht, Verzerrungen aufgrund subjektiver Willkür auszuschalten. Prozedurale Objektivität möchte das Erkenntnissubjekt als ,epistemologisches Risiko' ausschalten, indem es - was an der quantitativ-statistischen Variante prozeduraler Objektivität besonders deutlich wird - quasi-mechanische Prozeduren und Methoden vorschreibt, mit welchen die individuelle Urteilskraft und damit das vermeintliche Verzerrungsrisiko aufgrund persönlicher (unwissenschaftlicher) Präferenzen reduziert oder im Idealfall beseitigt werden sollen. Die prozedurale Objektivität möchte also das Fernziel einer korrespondierenden Darstellung erreichen, indem sie das Nahziel der Unparteilichkeit des Erkenntnis-

42 Vgl. Porter 1994, 197. Daher platziert Douglas die prozedurale Objektivität zurecht bei ihrer „objectivity ““, die sich mit den sozialen Prozessen beschäftigt, die zu Objektivität führen können (Douglas 2004, 461 f.).

43 Auf diese Unzulänglichkeit prozeduraler Objektivität hat Douglas hingewiesen $(2004,462)$. 
subjekts erzwingen möchte.

Die Kategorie der prozeduralen Objektivität steht demnach in einem dreifachen Abhängigkeitsverhältnis: erstens zur Objektivität als Unparteilichkeit, zweitens zur konsensualen Objektivität und drittens zum korrespondenztheoretischen Begriffskern, weil prozedurale Objektivität, selbst dann, wenn es ihr tatsächlich gelänge, subjektive Verzerrungen restlos auszuschalten, nur ein Mittel zu einer korrespondierenden Darstellung wäre.

\subsubsection{Objektivität als Unparteilichkeit}

Die Quintessenz prozeduraler Objektivität bestand darin, objektive Ergebnisse erreichen zu wollen, indem objektive Methoden angewandt werden, die gleichsam mechanisch den vermeintlich erkenntnisverzerrenden Spielraum an persönlicher Willkür des Historikers ausschalten sollen. Sie soll einen von der Persönlichkeit und der Perspektive des Historikers unverzerrten Zugang zu den reinen, unverfälschten ,Fakten' liefern. Prozedurale Objektivität verfolgt dieses Ziel, indem sie fachdisziplinär-konsensuell festgelegte Standards nutzt. Dagegen verbergen sich hinter der Objektivität als Unparteilichkeit (oder: Neutralität) keine inhaltlich exakt bestimmten Standards. Sie besitzt eher den Charakter einer wissenschaftsethischen Norm und damit eine partiell appellative Funktionsweise. Sie richtet sich an den Historiker als normativ ansprechbaren Akteur, der sich nach dieser (vagen) wissenschaftsethischen Norm zu richten hat und der nur im Fall eines groben Verstoßes gegen sie von der Gemeinschaft gerügt wird. Oftmals ist es gerade diese personalisierte Variante von Objektivität, die uns zuerst in den Sinn kommt, wenn die Rede auf Objektivität kommt. Sie ist in Gestalt von objektiven Schiedsrichtern, Richtern, Sachverständigen oder eben Historikern gewiss eine der am weitesten verbreiteten Anwendungen von „Objektivität"/,objektiv“".

Bevor die Abhängigkeitsfragen geklärt werden können, bedarf Unparteilichkeit als Objektivitätsvariante aber einer klärenden Erläuterung. 
$\mathrm{Zu}$ diesem Zweck sollen exemplarisch an der Diskussion historiographischer Neutralität durch Th. Haskell zwei verschiedene Auffassungen von Objektivität als Unparteilichkeit eingeführt werden.

Haskell unterscheidet zwischen der Forderung, sich selbst von eigenen Vorurteilen, Überzeugungen, Werten etc. zu befreien ,to become a perfectly passive and receptive mirror of external reality" (dem traditionellen positivistisch-historistischen Ideal) und einer Form der Neutralität, die eine vorläufige Distanzierung (,detachment") von der eigenen Perspektive beinhaltet, um auf diese Weise der Argumentation des Gegenübers vorurteilslos gerecht werden zu können. ${ }^{44}$ Distanzierung sei jedoch für sich genommen nicht Objektivität (wodurch er sich von der traditionellen Objektivität-ist-Neutralität-Position distanziert), sondern vielmehr eine Vorbedingung dafür, eigene Ziele, die im Zuge der Distanzierung nur vorläufig suspendiert worden sind, besser verfolgen zu können. Noch besser verfolgt werden sie, so Haskell, indem die eigene Sicht auf die Dinge dadurch bereichert wird, dass die gegnerische Position, die im Rahmen der Distanzierung auf ihre Qualität hin überprüft worden ist, in Teilen oder vollständig in die eigene Position integriert wird. Erst auf diese Weise werde ein objektiveres Ergebnis erzielt.

Haskells Darstellung führt also zunächst eine Variante von Objektivität als Unparteilichkeit ein, sc. die traditionelle Objektivitätskonzeption, deren Ideal die restlose ,Läuterung ${ }^{6}$ des Selbst von allen Voraussetzungen, Vorurteilen, Idealen, im Grunde von der eigenen Persönlichkeit ist. Diese Objektivitätskonzeption hält er für falsch. ${ }^{45}$ Wenn Objektivität aber nicht Neutra-

44 Haskell 1998, 148 und 150 (Hervorh. J. K.).

45 Für Beard und seine Zeitgenossen war das noch die entscheidende Fragestellung. Die Frage, ob und wie ein Historiker die eigene Persönlichkeit ,auslöschen' kann, um wahrhaft objektive Geschichte zu schreiben, hat die Kontroverse bestimmt. Vgl. Beard 1964, 317, 321 u. ö. Dabei hat sich der methodische Fehler eingeschlichen, die Unmöglichkeit historiographischer Objektivität (i. S. v. Neutralität) dadurch zu erweisen, dass den Vertretern dieser Position ihre Verwicklung in politische Auseinandersetzungen und weltanschauliche Kontroversen vorgehalten worden ist. Diese methodisch-argumentative Schieflage hat sich von Beard auf Novick 1988 , vererbt'. Sie wird von Haskell denn auch zurecht angegriffen. Haskell 1998, 153: „All this [die politische Parteinahme der Historiker bei gleichzei- 
lität i. S. v. Persönlichkeitsauslöschung ist, was kann dann mit Neutralität sinnvoll gemeint sein? Der Historiker müsse seine Emotionen und Voreingenommenheiten suspendieren können und zwar zu dem Zweck, die gegnerische Argumentation angemessen einschätzen und dann in die eigene Position integrieren zu können. Wenn Haskell selbst dies vielleicht auch nicht als genuine Objektivitätsauffassung ansehen möchte, so ist es doch naheliegend, diese Variante als eine weitere Möglichkeit anzusehen, Objektivität als Unparteilichkeit aufzufassen, und sie ein wenig genauer anzusehen. Diese Variante soll Unvoreingenommenheit genannt werden, sie beinhaltet nicht die Auslöschung der Persönlichkeit, ${ }^{46}$ soweit ist Haskell recht zu geben, sie muss aber ein wenig anders verstanden werden, als Haskell sie entwickelt.

Warum überhaupt soll ein Historiker, der seine eigenen politischen, wissenschaftlichen oder persönlichen Ziele besitzt, seine Ziele und die damit verbundenen Emotionen (vorläufig) suspendieren? Warum soll er, wenn er doch ein Ziel erreichen möchte, auf die unter Umständen besten Mittel zur Erreichung dieses Ziels verzichten, sprich, Beweise unterdrücken, Interpretationen ,übersehen' usf.? Der Grund ist, dass der Historiker mit einem solchen Verhalten lediglich Propaganda treiben würde, was dem Wissenschaftscharakter der Disziplin widerspräche. Ein Historiker muss, neben und über seinen sonstigen Zielen, in erster Linie das Ziel wissenschaftlicher Redlichkeit besitzen. Er muss einen Wert darin sehen, seine eigenen Voreingenommenheiten soweit es möglich ist, zu suspendieren bzw. sich von ihnen zu distanzieren und zwar in dem Sinn, dass er den Darstellungen und Argumentationen anderer mindestens denselben Grad an wissenschaftlicher Redlichkeit unterstellen sollte, wie er selbst für seine Arbeit rekla-

tiger Forderung nach wissenschaftlicher Objektivität] is presented [in Novick 1988] to the reader in a tone of bemused shock and wide-eyed dismay, as if by discovering connections between their scholarship and their likes and dislikes, we were catching the mighty with their pants down." Vgl. auch Daston 1998, $20 \mathrm{f}$. Damit soll nicht gesagt werden, dass sich die Argumentation Beards auf die ideologische Voreingenommenheit der Objektivisten beschränkt. Er prüft auch die philosophischen Thesen des positivistischen Objektivismus (1964, $323 \mathrm{ff}$.).

Haskell 1998, 149 f. 
miert. ${ }^{47}$ Wissenschaftliche Redlichkeit steht im Gegensatz zur bedingungslosen Verfolgung anderer, wissenschaftsexterner Ziele.

Die Begründung, warum ein Historiker sich provisorisch selbst beschränken soll, enthüllt die Zielsetzung, die hinter den Forderungen nach wissenschaftlicher Redlichkeit steht. Er möchte eine den untersuchten Gegenständen angemessene Darstellung erreichen. Nur wer sich unvoreingenommen den Argumenten, Beweisen, Interpretationen anderer öffnet, kann seine eigene Perspektive durch die des anderen anreichern und damit die eigene Darstellung vervollständigen. Bei Objektivität als Unparteilichkeit hier nicht i. S. der Auslöschung der Persönlichkeit, sondern einer mit wissenschaftlicher Redlichkeit einhergehenden Unvoreingenommenheit - handelt es sich demnach um eine derivative Objektivitätsvorstellung. Das eigentliche Ziel besteht darin, eine den Untersuchungsgegenständen angemessene Erkenntnis zu gewinnen, denn sonst wäre die unvoreingenommene Untersuchung mit dem Ziel der Integration von Perspektiven sinnlos, und ebenso sinnlos wäre die wissenschaftsethische Prioritisierung wissenschaftlicher Redlichkeit als Wert gegenüber anderen, mit ihm in Konkurrenz befindlichen, existentielleren Zielen des Historikers. Beide von Haskell gegeneinander abgewogene Formen der Unparteilichkeit (Neutralität), Auslöschung der Persönlichkeit und Unvoreingenommenheit, verfolgen folglich denselben Zweck: eine angemessene, sprich, korrespondierende Darstellungen des Geschehenen zu erreichen. Gleichgültig welche der beiden Varianten von Objektivität als Unparteilichkeit man zur Explikation dieser Objektvitätsvariante bevorzugt, muss man anerkennen, dass sie in einem derivativen Verhältnis zum Begriffskern stehen.

Darüber hinaus steht Unvoreingenommenheit ebenso wie prozedurale Objektivität zur fachdisziplinär-konsensualen Objektivität in einem Abhängigkeitsverhältnis, wenn auch nur soweit, als die jeweilige Einschätzung, wann ein Historiker gegen das Gebot der wissenschaftlichen Redlichkeit

47 Vgl. Mommsen 2000, 54 f.: ,The objectivity that is required of the historian in this light is not one of a detached historicism, but rather one of a critical reflection on one's own position, while paying due respect to existing alternative positions and the values on which these are based." 
verstoßen und sich als voreingenommen erwiesen hat oder nicht in ausreichendem Maß seine Persönlichkeit , ausgelöscht' hat, vom Konsens der Forschungsgemeinschaft bestimmt wird. Den Mitgliedern der scientific community kommt also die Aufgabe zu, mehr oder minder konsensuell die inhaltliche Bestimmung des vagen Normcharakters von Unparteilichkeit auszuhandeln.

\subsubsection{Konsensuale Objektivität}

Es bleiben damit noch die Abhängigkeiten der fachdisziplinär-konsensualen Objektivität ${ }^{48} \mathrm{zu}$ prüfen. Fachdisziplinär-konsensuale Objektivität ist auf den ersten Blick doppeldeutig. Teilweise gewinnt man den Eindruck, es handle sich bei fachdisziplinär-konsensualer Objektivität um die Vorstellung, dass objektive Erkenntnisse dann vorliegen, wenn ein Kollektiv darin übereinstimmt, objektive Erkenntnisse gefunden zu haben, das heißt, weil dieses Kollektiv Ergebnisse für objektiv erklärt, sind sie objektiv. Derart vereinfacht gelesen, ist das absurd, weil es im Grunde auf eine ,dekretorische $e^{6}$ Objektivität ${ }^{49}$, ein durch ein bloßes kollektives fiat zustande gekommenes Surrogat für Objektivität hinausliefe. Darüber hinaus handelte sich eine solche Auffassung das Problem ein, dass konkurrierende Gemeinschaften mit gleichem Recht ihre Ergebnisse als objektiv dekretieren könnten. Die Folge wäre ein Gruppenrelativismus, kein Objektivismus. So kann fachdisziplinär-konsensuale Objektivität von vornherein nicht plausibel sein.

Wenn sie vertretbar sein soll, dann nur in einer epistemischen Form. Objektive Ergebnisse sind nicht objektiv, weil sie zu solchen erklärt werden,

48 Vgl. die Erläuterungen zur ,disciplinary objectivity“ in Megill 1994b, 5-7 und auch Douglas' „objectivity ${ }_{3}$, die sich auf die sozialen Vorgänge konzentriert, die bei der Festlegung epistemologisch wichtiger Prozeduren und bei der Erreichung von Übereinstimmung stattfinden (dies. 2004, 456 und 461-465).

49 Acham nennt diese Form der konsensualen Objektivität ,einen konsensuellen Schein der Objektivität“" $(1977,395)$. 
sondern sie sind als objektiv erkennbar und zwar ausschließlich oder vornehmlich durch die dafür ausgebildeten ,Fachkreise.$^{50}$ Diese zweite Form ist trivial, denn wer, wenn nicht die ausgebildeten Experten, soll die Richtigkeit von Erkenntnissen im jeweiligen Bereich beurteilen können? Aufgewertet wird die konsensuale Objektivität denn auch durch Überlegungen, die zum Beispiel kommunikationstheoretische Standards einführen: Wenn nur lange genug von kompetenten Diskussionsteilnehmern erörtert worden ist, was ein objektives Ergebnis ist, dann wird das Ergebnis (mit hoher Wahrscheinlichkeit) objektiv sein. ${ }^{51}$

Welche exakte Lesart man der konsensualen Objektivität auch geben mag, ihr epistemologisches Ziel besteht aber darin, Erkenntnisse als mit den untersuchten Phänomenen korrespondierend ausweisen zu können. ${ }^{52}$ So ist etwa Bevirs Vorschlag ${ }^{53}$, Objektivität aus dem Vergleich verschiedener Interpretationen über Fakten hervorgehen zu lassen, über deren Wahrheit Konsens herrscht, nur dann sinnvoll, wenn das Endziel immer noch in der Korrespondenz der durch den Vergleich verbesserten Interpretationen besteht. Man hat mit konsensualer Objektivität gewiss einen wichtigen

50 Zu diesem Ansatz möchte ich auch den Vorschlag Hedingers (1977) rechnen, der ihn allerdings nicht als Objektivitätsvariante betrachtet, sondern vielmehr als eigenständiges Drittes zwischen Objektivität und Subjektivität. Für ihn erschöpft sich Objektivität in einem Abbildungsobjektivismus, den er ablehnt (365). Aus der Unmöglichkeit des Abbildungsobjektivismus folge aber noch nicht die Notwendigkeit des „Subjektivismus“, denn es gebe noch „,die Termini der ,intersubjektive[n] Allgemeingültigkeit' oder ,verbindliche[n] Prüfbarkeit “", die „,das Gewicht auf die Möglichkeit begründeter Übereinstimmung der Forscher“" lege (368). Die begründete Übereinkunft ist, so verstehe ich Hedinger, das Maß der Wissenschaftlichkeit. Seine Vorstellung von Objektivität ist zu eng und aus diesem Grund wird sein Vorschlag so gewertet, dass er eigentlich eine weitere Dimension der Objektivität offenlegt und nicht Objektivität verabschieden möchte. In seiner Beziehung zum eigentlichen Erkenntnisziel, sc. Korrespondenz, ist Hedingers Vorschlag ohnehin analog zur konsensualen Objektivitätsauffassung zu behandeln.

51 Diese Objektivitätsauffassung vertreten Appleby et al. 1994, 195 und passim.

52 Vgl. Patzig 1977, 324 f.

53 Bevir 1994, 333-337. 
Baustein in der Etablierung wissenschaftlicher Erkenntnis ${ }^{54}$, aber an der fundamentalen Rolle der absoluten Objektivität und damit am korrespondenztheoretischen Begriffskern ändert sich nichts. In dieser Hinsicht muss also auch die konsensuale Objektivität zu den derivativen Objektivitätsauffassungen gezählt werden.

Die bislang untersuchten Objektivitätsauffassungen, prozedurale Objektivität und Objektivität als Unparteilichkeit, haben sich jeweils als abhängig von der konsensualen Objektivität erwiesen. Der Erfolg der Methoden prozeduraler Objektivität, ihr Grad an tatsächlicher Verzerrungsfreiheit wird inhaltlich über den Konsens der scientific community eingeschätzt. Dass damit kein sicherer Anhalt für objektive Erkenntnisse gewonnen werden kann, dürfte jedem klar sein, der auch nur einigermaßen vom beständigen Methodenwandel in den Wissenschaften Kenntnis hat. So wird statistisch-quantitativen Methoden mal ein höherer, mal eine geringerer Objektivitätsgrad zuerkannt. Auch die ,Methode' der Quellenkritik, die als essentielle Voraussetzung für die Geschichtswissenschaft unverzichtbar ist (im Gegensatz zu zum Beispiel häufig bekämpften statistischen Ansätzen), ist nur insofern Anhaltspunkt für die Objektivität von Erkenntnissen, als die ,Fakten', die in den Quellen ,entdeckt' werden, inhaltlich vom fachdisziplinären Konsens partiell mitbestimmt und abgesegnet werden.

Analog kann Objektivität als Unparteilichkeit zumindest inhaltlich nur soweit bestimmt sein, wie sich ein fachdisziplinärer Konsens darüber etablieren kann, inwieweit die Methoden, Interpretationen und Faktengrundlagen eines Historikers tatsächlich unparteiischem, wissenschaftlich redlichem Forschen geschuldet ist oder inwieweit die notwendige Perspektivität

54 Setzt man prozedurale und konsensuale Objektivität in Beziehung - was hier nicht ausführlich getan werden kann, wenn es auch in den vorigen Abschnitten andeutungsweise geschehen ist -, dann kann man feststellen, dass konsensuale Objektivität sicherlich eine große Rolle bei der Überwindung der (hier nicht dargestellten) internen Schwierigkeiten der prozeduralen Objektivität spielen wird. Umgekehrt wird aber damit gerade das Verdienst prozeduraler Objektivität, sc. die Begrenzung willkürlicher Entscheidungen, auf der Ebene kollektiver Entscheidungen konterkariert, es sei denn, bestimmte Verfahrensanweisungen oder kommunikative Standards qualifizierten die kollektive Entscheidung als ,objektiv`, was wiederum eine disziplinär-konsensuale Entscheidung sein muss usf. 
in Parteilichkeit umgeschlagen ist. Prozedurale wie auch Objektivität als Unparteilichkeit sind demnach inhaltlich von fachdisziplinär-konsensualer Objektivität abhängig. Auch hier besteht demnach eine Abhängigkeitsbeziehung unter den verschiedenen Objektivitätsauffassungen.

\subsubsection{Das Komponentenmodell ist integrativ}

Das Komponentenmodell wurde als eine Weiterentwicklung der Koexistenzstrategie eingeführt. Wie die Koexistenzstrategie belässt es den gängigen und irreduziblen Objektivitätsauffassungen einen prominenten Platz innerhalb einer umfassenden Gesamtkonzeption von (historischer) Objektivität. Damit wird es dem Sprachgebrauch von „objektiv“/,Objektivität“ gerecht, der eine restlose Reduktion der diversen Objektivitätsauffassungen auf eine Definition (wie dies die Realdefinitionsstrategie vorsieht) so aussichtslos wie witzlos erscheinen lässt. Das Komponentenmodell geht jedoch über das bloße Konstatieren und das gewissermaßen parataktische Nebeneinanderstellen von Objektivitätsauffassungen hinaus, indem es einen integrierenden Begriffskern vorschlägt und darüber hinaus die von diesem Begriffskern abhängigen Objektivitätsauffassungen untereinander in Beziehung setzt (was ansatzweise gezeigt worden ist). Es ist demnach integrativ und zwar in dem Sinn, dass es die verschiedenen Objektivitätsauffassungen in ein Modell integriert, ohne dass eine der Auffassungen die andere vereinnahmt oder sie freischwebend über eine bloße „,begriffliche Kohärenz" zusammengehalten werden.

Der Begriffskern wie auch die derivativen Objektivitätsvarianten sind also in diesem Modell unverzichtbare und dabei integrierte respektive integrierende Komponenten eines vielschichtigen Konzepts. Das belegt unser alltäglicher wie unser wissenschaftlicher Sprachgebrauch. Sollten mit „objektiv“ lediglich die derivativen Objektivitätsauffassungen (oder eine unter ihnen) gemeint sein, dann müsste es möglich sein, dass auch falsche Aussagen als objektiv bezeichnet werden könnten, weil wir trotz erprobter, 
objektiver Methoden, unserer größtmöglichen Anstrengung wissenschaftlich redlich vorzugehen und auch des bestens abgesicherten Konsensus der Wissenschaftsgemeinschaft immer wieder falsche Ergebnisse erzielen. Wir bezeichnen aber falsche Aussagen (i. e. den Tatsachen nicht korrespondierende Aussagen) nicht als objektiv, ebenso wenig wie wir eine historische Darstellung als objektiv bezeichnen würden, die zwar methodisch einwandfrei erarbeitet worden ist, aber dennoch nicht mit den Tatsachen korrespondiert. Wer also (aufgrund welcher objektiver Methoden auch immer) zur Überzeugung, dass $p$ kommt, der kann, wenn sich herausgestellt hat, dass diese Überzeugung falsch ist, nicht behaupten, die objektive Erkenntnis, dass $p$, zu besitzen. Und umgekehrt kann eine lediglich korrespondierende Darstellung, die auf welchem wundersamen Wege auch immer gewonnen worden ist, nicht als objektiv bezeichnet werden, weil im Sprachgebrauch von „objektiv“/,Objektivität“ auch die Form der Erkenntnisgewinnung eine Rolle spielt.

Diesem Befund aus dem Sprachgebrauch kann nur ein Objektivitätsmodell gerecht werden, das, wie das hier vorgeschlagene Komponentenmodell, die integrative Leistung vollbringt, mehrere irreduzible Objektivitätsauffassungen in einem Modell zu vereinen, statt eine bloße Koexistenz unter einem unverbindlichen Sammelbegriff vorzusehen oder gar eine Realdefinitionsstrategie zu verfolgen. Wie die vorangegangenen Abschnitte gezeigt haben, integriert der korrespondenztheoretische Begriffskern (i. e. die absolute Objektivität) die übrigen drei Objektivitätsauffassungen über das Abhängigkeitsverhältnis, in dem sie qua Mittel zu dem Zweck stehen, eine Korrespondenz zwischen Darstellung und Dargestelltem zu erreichen. Dass daneben weitere (nur angedeutete) systematische Beziehungen zwischen den derivativen Objektivitätsauffassungen bestehen, belegt darüber hinaus, dass ein Erforschen des Objektivitätsbegriffs anhand eines Komponentenmodells einen adäquateren Ansatz darstellt als ein bloßes Koexistenzmodell.

Dass sich die meisten Arbeiten zur (historischen) Objektivität auf eine oder einige wenige Objektivitätsauffassung(en) konzentrieren und dabei andere Auffassungen unerwähnt beiseite lassen (sich wohlgemerkt also weder für noch gegen ihren Status als genuine Objektivitätsauffassungen aus- 
sprechen), ist weniger ein Indiz dafür, dass das Komponentenmodell als integratives Modell verfehlt ist, sondern eher eine Folge einer zwangsläufigen argumentativen Fokussierung, ohne die keine Arbeit zur (historischen) Objektivität zu einem abgeschlossenen Ganzen werden könnte. Eine Fokussierung auf eine bestimmte Objektivitätsauffassung, die zu analytischargumentativen Zwecken aus der Integrität des Gesamtmodells ausgegliedert wird, ist sinnvoll und berechtigt, weil nicht jeder Untersuchungsgegenstand und jede Argumentation ein integratives Objektivitätsmodell wie das Komponentenmodell ständig in seiner Vollständigkeit im Blick zu behalten braucht.

Auf welche Objektivitätsauffassung fokussiert wird, ergibt sich in erster Linie durch die jeweilige Form von epistemologischem Skeptizismus, der sich Objektivitätsansprüche gegenüber sehen können. Wer davon überzeugt ist, dass der ,menschlichen Faktor' immer Verzerrungen in die Erkenntnis bringt, der wird versuchen, ihn durch objektive Methoden wie Quantifikation und Statistik auszuschalten; wer der Ansicht ist, Werte seien immer verzerrend, wird normativ festlegen wollen, dass Werte in der Wissenschaft nichts zu suchen haben usf. Auch in der vorliegenden Arbeit wird eine solche Fokussierung vorgenommen werden müssen und zwar eine Fokussierung auf die korrespondenztheoretisch explizierte absolute Objektivität. Das bedeutet aber nicht, dass damit die integrative Qualität des Komponentenmodells desavouiert wird, es handelt sich vielmehr um eine Einschränkung aus einer Zweckmäßigkeitserwägung heraus. 


\subsection{Objektivität und Standortgebundenheit}

Auch die Fokussierung der weiteren Ausführungen auf die Diskussion um die korrespondenztheoretisch explizierte absolute Objektivität ist einem wissenschafts- und erkenntnistheoretischen Skeptizismus geschuldet. Diese Form des Skeptizismus rüttelt wie keine zweite am fundamentalen Selbstbewusstsein der Geschichtswissenschaft. Dem Anspruch der Geschichtswissenschaft auf die Objektivität ihrer Erkenntnis steht das Schlagwort von der Standortgebundenheit historischer Erkenntnis als ein geschichtstheoretischer Gemeinplatz gegenüber. ${ }^{55}$ Wo historische Erkenntnis die Vergangenheit beschreiben soll, ,wie es eigentlich gewesen“"56, wird von ihr gleichzeitig gesagt, sie sei unentwirrbar oder notwendig vom Standort, das

55 Hedinger 1977 definiert „Standortgebundenheit“ folgendermaßen: „Sie [sc. die These der Standortgebundenheit] besagt, da $\beta$ sich in einer historischen Aussage unvermeidlich und irreversibel Inhaltsbestandteile finden, die nicht den geschichtlichen Gegenstand, sondern den konkreten Standort des Historikers kennzeichnen. Dazu gehören Vorverständnis, Sinnbestimmungen, (,erkenntnisleitende`) Interessen, geschichtsphilosophisch-anthropologische Überzeugungen usw. der Gegenwart des Historikers [...]. Alle historischen Aussagen stehen ,gleichsam mit einem Bein darin'. Und nicht nur Aussagen, auch Fragen, Begriffe, empirisch fundierte Theorien, Modelle, Methoden des Historikers sind dann durch die - sich zudem ständig wandelnde - Perspektive seines Standorts geprägt.“ (ebd., 368 f.) So auch Rüsen: „Historical knowledge can neither be gained nor presented without a perspective linked to the situation of the provider or the target audience.“ $(2000,59)$ Vgl. auch Lorenz 1997, Mommsen 1977 , Rüsen 1983, 2002a und 2002b.

56 Dieses Ranke-Zitat darf in keiner Arbeit über historiographische Objektivität fehlen, es sei daher auch hier in voller Länge wiedergegeben: „Man hat der Historie das Amt, die Vergangenheit zu richten, die Mitwelt zum Nutzen zukünftiger Jahre zu belehren, beigemessen: so hoher Ämter unterwindet sich gegenwärtiger Versuch nicht: er will bloß zeigen, wie es eigentlich gewesen.“ (L. v. Ranke „Geschichte der romanischen und germanischen Völker", SW Bd. 33/34, Seite VII) Vgl. die sorgfältige Analyse Vierhaus' 1977, die drei Säulen identifiziert, auf denen Rankes Objektivitätskonzeption ruht (und damit für Generationen von Historikern die maßgebliche Objektivitätskonzeption war): Quellenstudium, Unparteilichkeit und „objektive Darstellung“ der Erkenntnisse. (Ebd., 65) 
heißt von der Perspektive des jeweiligen Historikers abhängig. Die Geschichtstheorie sieht sich damit in der Position, mit zwei auf den ersten Blick nur schwer vereinbaren, wenn auch nicht strikt kontradiktorischen theoretischen Grundhaltungen zurande kommen zu müssen.

Ein sich im Lauf des späten 18. Jahrhunderts entwickelnder Wunsch nach Vereinbarung dieser Unvereinbarkeit ist geistesgeschichtlich analysiert worden als ein Schwanken zwischen einer Orientierung an einem vormodernen „,naiven Realismus“ einerseits, mit seinen Metaphern der „nackten Wahrheit“, dem „Wie-es-eigentlich-gewesen“, der Geschichte als „speculum vitae humanae“ und andererseits der epistemologisch notwendigen Perspektivität, die durch die Weiterentwicklung des naiv-realistischen optischen Metaphernfeldes (i. e. des Spiegelns, Abbildens, Sehens etc.), nahegelegt wurde. R. Koselleck hat diese Analyse prägnant in eine knappe Geschichte der Geschichtstheorie eingezeichnet. ${ }^{57}$ Dieses Schwanken kann also kaum als ein geschichtstheoretisches Novum betrachtet werden, gleichwohl scheint die Geschichtstheorie ${ }^{58}$ noch immer, das zeigt nicht zuletzt Kosellecks Beitrag, schwer an dieser Konstellation zu tragen, die er als „erkenntnistheoretisches Dilemma“ ${ }^{\text {bezeichnet. }}{ }^{59} \mathrm{Um}$ ein Dilemma in

57 Koselleck 1979. Besonders betont wird die bahnbrechende Einsicht Chladenius', dass die Abhängigkeit historischer Erkenntnis vom „Sehepunct“ (Chladenius) des jeweiligen Historikers, die epistemologische Voraussetzung von Erkenntnis überhaupt ist und dabei nicht mit Parteilichkeit verwechselt werden darf (Koselleck 1979, 184-188).

58 Natürlich gilt dies nicht nur für die Geschichtstheorie. S. Harding hat nachzuweisen versucht, dass eine Objektivitätsauffassung, die sich am Ideal strikter Neutralität des Forschers orientiert und die darauf hinausläuft, sämtliche sozio-kulturellen Einflüsse auf die Forschung ausschalten zu wollen, mitunter dem Erkenntnisgewinn abträglich war; was sowohl für die sozial- wie auch für die naturwissenschaftliche Forschung gelte (dies. 2003, 170 u. ö.).

59 Man könnte selbstverständlich auch eine stoische Gelassenheit an den Tag legen. Vgl. Murpheys lakonischen Kommentar zur vermeintlich ,tödlichen' Gefahr, die der historischen Erkenntnis drohe, wenn man der Erkenntnis zustimmte, Teil der Interpretation historischer Beweise seien immer die eigenen Voraussetzungen: „That we are creatures who live in time, that we inherit a cultural tradition, and that we approach the interpretation of texts, and everything else, with certain ideas already in mind, no one denies. But so what? The same claims hold with re- 
einem engen Sinn handelt es sich zwar nicht, eher um zwei epistemologisch-wissenschaftstheoretische Fundamentalpositionen mit nicht leicht vereinbaren Gehalten, dennoch steckt in diesem vermeintlichen „Dilemma" der Kern des Objektivitätsproblems in der Geschichtswissenschaft. Daher wird die weitere Untersuchung auf die Vereinbarung korrespondenztheoretisch zentrierter historischer Objektivität mit prima facie korrespondenzskeptischer Standortgebundenheit fokussiert.

\subsubsection{Korrespondenzcharakter und Standortgebundenheit}

Der Anschein eines Dilemmas drängt sich nachdrücklich auf, weil jeder der beiden widerstreitenden geschichtstheoretischen Grundpositionen eine große Plausibilität zugestanden werden muss. Von historischen Darstellungen wird gemeinhin erwartet, dass sie darstellen, was sich in der Vergangenheit zugetragen hat. Sie sollen uns darüber aufklären, wo unsere soziokulturellen Wurzeln liegen, welche Kontinuitäten uns mit unseren Vergangenheit verbinden oder wo die Verbindung abgerissen ist, wo wir uns mit den Ruhmestaten unserer Vorfahren identifizieren können oder uns für deren Schandtaten schämen müssen; Geschichtsschreibung legt, zusammen mit anderen identitätsstiftenden Genres wie dem Mythos, der Legende, der Hagiographie o. ä., gar erst fest, wer zu den gegenwärtigen und den vergangenen Gemeinschaften zugehört, denen wir angehören und was dies für Gemeinschaften sind, deren Mitglieder wir sind.

Der Beitrag der Geschichte, der sie spezifisch bezeichnet und auszeichnet und der von keiner der ihr verwandten Genres retrospektiver sozio-kultureller Selbstbestimmung wie zum Beispiel dem Mythos oder dem Epos übernommen werden kann, besteht darin, dass sie der jeweiligen Gemeinschaft ein ,korrektes' Bild davon gibt, wie die Vergangenheit aussieht, in der sie verwurzelt ist. Dieser abspiegelnde Beitrag, der ebenso im Common sense wie auch in der Geschichtswissenschaft selbst intuitiv mit „Ge- 
schichte" verbunden wird, kommt nicht ohne Korrespondenz zwischen den Ereignissen und ihren Schilderungen und Analysen aus. Geschichte gibt die Tatsachen der Vergangenheit korrespondierend wieder - sie besitzt einen Korrespondenzcharakter. ${ }^{60}$ Auf diese Weise würde jeder, der nach einer möglichst allgemeinen Bestimmung der Funktion oder Aufgabe der Geschichte (i. S. v. historia rerum gestarum) gefragt wird, antworten - es sei denn, er ist durch geschichtstheoretisch-philosophische Kenntnisse bereits vom Zweifel an eine solche schlichte Auffassung, angekränkelt' und kann daher diese Antwort nicht mehr uneingeschränkt geben.

So steht dieser Common-sense-Vorstellung vom Korrespondenzcharakter der Geschichte ein erkenntnistheoretischer Skeptizismus gegenüber, der sich aufgrund einer ganzen Reihe von theoretischen Einsichten in das Wesen der (wissenschaftlichen) Geschichtsschreibung in eine Gegenposition $\mathrm{zu}$ dieser Vorstellung stellt. Die notwendige Standortgebundenheit oder Perspektivität historischer Erkenntnisse problematisiert den Common-sense-Anspruch auf ein Abspiegelungsverhältnis, das vermeintlich zwischen der Vergangenheit und ihrer historischen Darstellung besteht. Damit ist nicht gesagt, dass Standortgebundenheit jegliches Korrespondenzverhältnis ausschließt. Wer die These von der Standortgebundenheit historischer Erkenntnis ernst nimmt, ist lediglich dazu gezwungen, ein simples, positivistisches Abspiegelungsverhältnis oder genauer: ein erkenntnis- und wissenschaftstheoretisches Programm aufzugeben, das zu einem feststehenden (eben: „objektiven“) Bild der Vergangenheit führen soll, „wie es eigentlich gewesen". Was er darüber hinaus für epistemologisch-wissenschaftstheoretische Positionen vertritt, ist eine weitere Frage, die noch beschäftigen wird. Die Standortgebundenheit zu betonen, bedeutet also zunächst nur einmal, sich von einer positivistischen Auffassung historischer Erkenntnisgewinnung abzugrenzen, ohne aber die Gültigkeit des Korrespondenzcharakters der Geschichte insgesamt zu verwerfen. Das bedeutet, dass der eigentliche Widerstreit nur zwischen einem epistemologisch-wissenschaftst-

60 Topolski nennt dies den „faktographischen Aspekt von Geschichte“ (ders. 1987, 210). Die Einteilung in faktuale und fiktionale Narrationen ist eine geläufige Grundunterscheidung in der Erzähltheorie (vgl. Martinez/Scheffel 2003, Kap. I,1 „Faktuales und fiktionales Erzählen“, 9-19). 
heoretischen Programm und dessen Auffassung von vermeintlich verzerrenden Einflüssen auf objektive Darstellungen entsteht, nicht aber zwischen Objektivität und Standortgebundenheit als solchen.

\subsubsection{Die Mitte zwischen zwei Extremen}

Wie verhält sich die Geschichtstheorie angesichts dieser beiden widerstreitenden Pole? Ohne hier bereits weitere inhaltliche Bestimmungen geben zu wollen, können grundsätzlich drei Lösungswege, auf denen mit dem Widerstreit Schluss gemacht werden soll, schematisch skizziert werden.

(1) Man kann den Stier bei den Hörnern packen, indem man auf die Standortgebundenheit pocht, sie ausweitet und konsequent den Korrespondenzcharakter der Geschichte vollends negiert. In diesem Fall könnte von einem subjektivistischen Lösungsversuch gesprochen werden. Er ist insbesondere von Theoretikern postmoderner Couleur in etlichen Varianten unternommen worden.

(2) Auf der anderen Seite kann man sich restlos zum anderen Extrem bekennen. Aus dem Korrespondenzcharakter der Geschichte wird ein positivistisches Programm abgeleitet, das sich von jeglichem perspektivischen Moment freihalten möchte, weil Perspektivität als objektivitätsgefährdend und damit wissenschaftsgefährdend angesehen wird.

(3) Zwischen diesen beiden Extremen liegt die Vereinbarung des Korrespondenzcharakters mit der Standortgebundenheit historischer Erkenntnis als ,third way“. Auf diesem Mittelweg zwischen den Extremen hindurch zu navigieren, wird hier mittels eines historischen Konstruktionismus versucht. Was auch auf diesem ,third way“ genauer liegen mag, wird einen simplen Positivismus nicht aber den Korrespondenzcharakter negieren.

$\mathrm{Ob}$ die postmoderne Schlussfolgerung von der notwendigen Standortgebundenheit historischer Erkenntnis auf die Verabschiedung historischer Objektivität plausibel vertretbar ist, wird im nächsten Kapitel erörtert wer- 
den müssen. Dort wird die Untersuchung auf den postmodern-narrativistischen Argumentationsstrang eingegrenzt werden, der seinerseits exemplarisch an der Theorie Frank Ankersmits evaluiert werden wird. Das Ziel der argumentativen Auseinandersetzung mit dem postmodernen Narrativismus besteht darin, seinen Anspruch auf Verabschiedung von historischer Objektivität und damit sein Votum für den subjektivistischen Lösungsweg (1) zurückzuweisen. Dabei werden sich, gewissermaßen als erfreulicher Nebeneffekt, einige theoretische Bereicherungen für die Ausarbeitung einer eigenen Position im letzten Kapitel ergeben.

Bevor jedoch eine eigene Position in Grundzügen ausformuliert werden kann, müssen auch die Möglichkeiten und Limitierungen des positivistischen Lösungswegs (2) betrachtet werden. Er teilt mit dem ,third way“ die Vorstellung von einer engen, unaufgebbaren Beziehung zwischen Objektivität und Wissenschaftlichkeit der Geschichtswissenschaft. Wo sich jedoch der Weg des Lösungsvorschlags (3) von dem des Positivismus trennt, ist in der Beurteilung der Standortgebundenheit historischer Darstellungen. Der Positivismus vertritt eine Position, die davon ausgeht, dass objektive Erkenntnis eine perspektivenlose, ,unverzerrte Abspiegelung ist, die durch keinerlei Beitrag des Erkenntnissubjekts „,kontaminiert“ werden dürfe, während dagegen der ,third way“, in einem positivistischen epistemologischen Katalog ein unnötig rigoristisches Erkenntnisprogramm sieht.

Damit vertritt der Lösungsweg (3) ein eigenes Programm, das ihn zwischen die beiden Extreme der strikten Objektivitätsverabschiedung auf der einen Seite und des Objektivitätsrigorismus auf der anderen Seite platziert. Die Fundamente dieses Programms sind die notwendige Standortgebundenheit historischer Erkenntnis, bei gleichzeitiger Korrespondenz derselben mit der beschriebenen Vergangenheit. Hinzukommt die Ansicht, historische Darstellungen seien wesentlich narrativ. Dies ist ihm und dem postmodernen Narrativismus gemeinsam. Doch während ersterer den wesentlich narrativen Charakters der Geschichte mit ihrem Objektivitätsanspruch vereinbar erachtet, glaubt der postmoderne Narrativismus aus dem wesentlich narrativen Charakter historischer Darstellungen, die Verabschiedung historischer Objektivität ableiten zu können. Inwiefern der postmodernnarrativistische Anspruch auf Objektivitätsverabschiedung plausibel ist, 
wird im folgenden untersucht werden. Danach soll erst ein minimalistischer Narrativismus als Gegenentwurf in Grundzügen vorgestellt werden, der die Basis für die Vereinbarung von Objektivität mit Standortgebundenheit und Narrativität abgibt. 
